\title{
Dynamical measure and field theory models free of the cosmological constant problem
}

\author{
E. I. Guendelman* and A. B. Kaganovich ${ }^{\dagger}$ \\ Physics Department, Ben Gurion University of the Negev, Beer Sheva 84105, Israel
}

\begin{abstract}
We study field theory models in the context of a gravitational theory without the cosmological constant problem (CCP). The theory is based on the requirement that the measure of integration in the action is not necessarily $\sqrt{-g}$ but it is determined dynamically through additional degrees of freedom, like four scalar fields $\varphi_{a}$. We study three possibilities for the general structure of the theory: (A) The total action has the form $S=\int \Phi L d^{4} x$ where the measure $\Phi$ is built from the scalars $\varphi_{a}$ in such a way that the transformation $L \rightarrow L+$ const does not effect equations of motion. Then an infinite dimensional shifts group of the measure fields (SGMF) $\varphi_{a}$ by arbitrary functions of the Lagrangian density $L, \quad \varphi_{a} \rightarrow \varphi_{a}+f_{a}(L)$, is recognized as the symmetry group of the action up to an integral of a total divergence. (B) The total action has the form $S=S_{1}+S_{2}, S_{1}=\int \Phi L_{1} d^{4} x$, $S_{2}=\int \sqrt{-g} L_{2} d^{4} x$ which is the only model different from (A) and invariant under SGMF (but now with $\left.f_{a}=f_{a}\left(L_{1}\right)\right)$. Similarly, now only $S_{1}$ satisfies the requirement that the transformation $L_{1} \rightarrow L_{1}+$ const does not effect equations of motion. Both in the case (A) and in the case (B) it is assumed that $L, L_{1}, L_{2}$ do not depend on $\varphi_{a}$. (C) The action includes a term which breaks the SGMF symmetry. It is shown that in the first order formalism, a constraint appears which allows us to solve the scalar field related to the dynamical measure degrees of freedom, in terms of matter fields. The remarkable feature of models discussed in this paper is that for all cases ((A), (B) and (C)), after the change of variables to the conformal Einstein frame, the classical field equations take exactly the form of General Relativity (GR). Therefore the models are free from the well known problem of the usual scalar-tensor theories in what is concerned with the classical GR tests. The only difference of the field equations in the Einstein frame from the canonical equations of the selfconsistent system of Einstein's gravity and matter fields, is the appearance of the effective scalar field potential which vanishes in a true vacuum state (TVS) without fine tuning in cases (A) and (B). To illustrate how the theory works, we present a few explicit field theory models where it is possible to combine the solution of the cosmological constant problem (CCP) with: 1) possibility for inflationary scenario; 2) spontaneously broken gauge unified theories (including fermions). In the case (C), the breaking of the SGMF symmetry induces a nonzero energy density for the TVS. When considering only a linear potential for a scalar field $\phi$ in $S_{1}$, the continuous symmetry $\phi \rightarrow \phi+$ const is respected. Surprisingly, in this case SSB takes place while no massless ("Goldstone") boson appears. We discuss the role of the SGMF symmetry for quantization and the possible connection of this theory with theories of extended objects.

PACS number(s): 11.15.Ex, 98.80.Cq, 12.10.Dm, 04.90.+e
\end{abstract}

${ }^{*}$ GUENDEL@BGUmail.BGU.AC.IL

${ }^{\dagger}$ ALEXK@BGUmail.BGU.AC.IL 


\section{INTRODUCTION}

The cosmological constant problem in the context of general relativity (GR) can be explained as follows. In GR one can introduce such a constant or one may set it to zero. The problem is that after further investigation of elementary particle theory, we discover new phenomena like radiative corrections, the existence of condensates, etc. each of which contributes to the vacuum energy. In order to have a resulting zero or extremely small cosmological constant as required by observations of the present day universe, one would have to carefully fine tune parameters in the Lagrangian so that all of these contributions more or less exactly cancel. This question has captured the attention of many authors because, among other things, it could be a serious indication that something fundamental has been missed in our standard way of thinking about field theory and the way it must couple to gravity. For a review of this problem see [四].

The situation is made even more serious if one believes in the existence of an inflationary phase for the early universe, where the vacuum energy plays an essential role. The question is then: what is so special about the present vacuum state which was not present in the early universe? In this paper we are going to give an answer this question.

As it is well known, in nongravitational physics the origin from which we measure energy is not important. For example in nonrelativistic mechanics a shift in the potential $V \rightarrow V+$ constant does not lead to any consequence in the equations of motion. In the GR the situation changes dramatically. There all the energy density, including the origin from which we measure it, affects the gravitational dynamics.

This is quite apparent when GR is formulated from a variational approach. There the action is

$$
\begin{gathered}
S=\int \sqrt{-g} L d^{4} x \\
L=-\frac{1}{\kappa} R(g)+L_{m}
\end{gathered}
$$

where $\kappa=16 \pi G, R(g)$ is the Riemannian scalar curvature of the 4-dimensional space-time with metric $g_{\mu \nu}, g \equiv$ $\operatorname{Det}\left(g_{\mu \nu}\right)$ and $L_{m}$ is the matter Lagrangian density. It is apparent now that the shift of the Lagrangian density $L$, $L \rightarrow L+C, C=$ const is not a symmetry of the action (11). Instead, it leads to an additional piece in the action of the form $C \int \sqrt{-g} d^{4} x$ which contributes to the equations of motion and in particular generates a so called "cosmological constant term" in the equations of the gravitational field.

In Refs. [2]- 5] an approach has been developed where the cosmological constant problem is treated as the absence of gravitational effects of a possible constant part of the Lagrangian density. The basic idea is that the measure of integration in the action principle is not necessarily $\sqrt{-g}$ but it is allowed to "float" and to be determined dynamically through additional degrees of freedom. In other words the floating measure is not from first principles related to $g_{\mu \nu}$, although relevant equations will in general allow to solve for the new measure in terms of other fields of the theory $\left(g_{\mu \nu}\right.$ and matter fields). This theory is based on the demand that such measure respects the principle of non gravitating vacuum energy (NGVE principle) which states that the Lagrangian density $L$ can be changed to $L+$ constant without affecting the dynamics. This requirement is imposed in order to offer a new approach for the solution of the cosmological constant problem. Concerning the theories based on the NGVE principle we will refer to them as NGVE - theories.

The invariance $L \longrightarrow L+$ constant for the action is achieved if the measure of integration in the action is a total derivative, so that to an infinitesimal hypercube in 4-dimensional space-time $x_{0}^{\mu} \leq x^{\mu} \leq x_{0}^{\mu}+d x^{\mu}, \mu=0,1,2,3$ we associate a volume element $d V$ which is: (i) an exact differential, (ii) it is proportional to $d^{4} x$ and (iii) $d V$ is a general coordinate invariant. The usual choice, $\sqrt{-g} d^{4} x$ does not satisfy condition (i).

The conditions (i)-(iii) are satisfied [2], [3] if the measure corresponds to the integration in the space of the four scalar fields $\varphi_{a},(a=1,2,3,4)$, that is

$$
d V=d \varphi_{1} \wedge d \varphi_{2} \wedge d \varphi_{3} \wedge d \varphi_{4} \equiv \frac{\Phi}{4 !} d^{4} x
$$

where

$$
\Phi \equiv \varepsilon_{a_{1} a_{2} a_{3} a_{4}} \varepsilon^{\mu \nu \lambda \sigma}\left(\partial_{\mu} \varphi_{a_{1}}\right)\left(\partial_{\nu} \varphi_{a_{2}}\right)\left(\partial_{\lambda} \varphi_{a_{3}}\right)\left(\partial_{\sigma} \varphi_{a_{4}}\right) .
$$

is a measure independent of $g_{\mu \nu}$ as opposed to the case of GR. In what follows we will call $\Phi$ the dynamical measure since its value in realistic cases is dynamically determined in terms of the other fields of the theory through the equations of motion as we will see. We will use also the terms the "dynamical" volume element or the volume element 
of the "dynamical space - time" for the expression $\Phi d^{4} x$ since according to Eq. (3), it is actually the volume element of the internal space of fields $\varphi_{a}$ and in the action it will take the place of the space - time volume element of GR.

Notice that the dynamical measure (4) is a particular realization of the NGVE-principle (for other possible realization see Refs. 近, [5]). For additional discussion of the geometrical meaning of this realization of the measure see Ref. [6]

We will study three possibilities for the general structure of the action:

(A) The most straightforward and complete realization of the NGVE principle (the so called strong NGVE principle) is a theory where the total action is just defined as follows

$$
S=\int L \Phi d^{4} x
$$

where $L$ is a total Lagrangian density. We assume in what follows that $L$ does not contain explicitly the measure fields, that is the fields $\varphi_{a}$ by means of which $\Phi$ is defined.

Introducing independent degrees of freedom related to the dynamical measure we arrive naturally at a conception that degrees of freedom associated with all possible geometrical objects (like metric, connection and measure) that appear, should be considered as independent ones. This is why we expect that the first order formalism, where the affine connection is not assumed to be the Christoffel coefficients in general should be preferable to the second order formalism where this assumption is made.

In fact, it is found [5], [7], [8], [9] that the NGVE theory in the context of the first order formalism does indeed provide:

1) A possibility to construct models with the same number of degrees of freedom as in GR and with the same structure of equations after a suitable change of variables. In particular, it means that in the contrast to the usual scalar - tensor theories, a conformal frame exists where both the gravitational constant and all masses are constant simultaneously. It implies also that gravitational radiation for example will be the same as that in GR. By the same reason, in the true vacuum, the only spherically symmetrical solution is Schwarzschild. Therefore, the classical GR tests are guaranteed to work out correctly.

2) A solution of the cosmological constant problem.

We have to emphasize that this is not the case when using the second order formalism [2], [3].

The simplest example [4], [5] (see also Sec.IIC) where these ideas can be tested is that of a matter Lagrangian described by a single scalar field with a nontrivial potential. In this case the variational principle leads to a constraint which implies the vanishing of the effective vacuum energy in any possible allowed configuration of the scalar field. These allowed configurations are however constant values at the extrema of the scalar field potential and an integration constant that results from the equations of motion has the effect of exactly canceling the value of the potential at these points. So, the scalar field is forced to be a constant and hence the theory has no nontrivial dynamics for the scalar field.

In this case the measure (4) is not determined by the equations of motion. In fact a local symmetry (called "Local Einstein Symmetry" (LES)) exists which allows us to choose the measure $\Phi$ to be of whatever we want. In particular $\Phi=\sqrt{-g}$ can be chosen and in this case the theory coincides in the vacuum with GR with $\Lambda=0$.

A richer structure is obtained if a four index field strength which derives from a three index potential is allowed in the theory as it is demonstrated in Appendixes C and D and in Sec.IID (see also Refs. [5], [7] - 9]). The introduction of this term breaks the LES mentioned above. In this case, the constraint that the theory provides, allows to solve for the measure $\Phi$ in terms of $\sqrt{-g}$ and the matter fields of the theory. It turns out that by the use of a conformal transformation the equations can be written in the form of the Einstein theory equations where the resulting particle interactions and potentials are replaced by effective ones. Since the picture in these new variables has the structure of the Einstein's GR we will call this conformal frame the Einstein frame and we will refer to this picture as "Einstein picture".

Then the theory which contains a scalar field and a four index field strength shows a remarkable feature: the effective potential of the scalar field that one obtains in the Einstein picture is such that generally allows for an inflationary phase which evolves at a later stage, without fine tuning, to a vacuum of the theory with zero cosmological constant [5], [7]- [9].

It is interesting to notice the existence of the symmetry of the classical equations of motion under the global transformation $\Phi \rightarrow \alpha \Phi, \alpha=$ constant (which can be obtained by individual global rescalings of the $\varphi_{a}$ 's like $\left.\varphi_{a} \rightarrow \lambda^{1 / 4} \varphi_{a}\right)$. In the Einstein frame this symmetry includes a global scale transformation of the metric. The expectation value of 4 -index field strength spontaneously breaks this scaling symmetry without generating a massless dilaton.

The 4-index field strength also allows for a Maxwell-type dynamics of gauge fields [8], [9] and of massive fermions. For illustration how the theory works, the model which provides a possibility for an explicit construction of unified 
gauge theory $(S U(2) \times U(1)$ as an example) based on these ideas and keeping all the above mentioned advantages, is presented in Sec.V.

(B) It is possible to check (see [2] and Sec. IIA) that the action (5) respects (up to an integral of a total divergence) the infinite dimensional group of shifts of the measure fields $\varphi_{a}$ (SGMF)

$$
\varphi_{a} \rightarrow \varphi_{a}+f_{a}(L)
$$

where $f_{a}(L)$ is an arbitrary differentiable function of the total Lagrangian density $L$. Such symmetry in general represents a nontrivial mixing between the measure fields $\varphi_{a}$ and the matter and gravitational fields (through L). As it was mentioned in Ref. [2], this symmetry prevents the appearance of terms of the form $f(\chi) \Phi$ in the effective action (where quantum corrections are taken into account) with the single possible exception of $f(\chi)=c / \chi$ where a scalar $c$ is $\chi$ independent. This is because in this last case the term $f(\chi) \Phi=c \sqrt{-g}$ is $\varphi_{a}$ independent. This possibility gives rise to the cosmological constant term in the action while the symmetry (6) is maintained. This can be generalized to possible contributions of the form $\int L_{2} \sqrt{-g} d^{4} x$ where $L_{2}$ is $\varphi_{a}$ independent function of matter fields and gravity if radiative corrections generate a term $f(\chi) \Phi$ with $f(\chi)=L_{2} / \chi$.

So, let us consider an action which consists of two terms

$$
\begin{aligned}
S & =S_{1}+S_{2} \\
S_{1} & =\int L_{1} \Phi d^{4} x \\
S_{2} & =\int L_{2} \sqrt{-g} d^{4} x
\end{aligned}
$$

Now only $S_{1}$ satisfies the requirement that the transformation $L_{1} \rightarrow L_{1}+$ const does not effect equations of motion, which is a somewhat weaker version of the NGVE principle. In this case, the SGMF symmetry transformation (6) is replaced by

$$
\varphi_{a} \rightarrow \varphi_{a}+f_{a}\left(L_{1}\right)
$$

The constraint which appears again in the first order formalism, allows now to solve the measure $\Phi$ in terms of $\sqrt{-g}$ and the matter fields of the theory without introducing the four index field strength (see Sec. III).

In scalar field models with potentials entering in $S_{1}$ and $S_{2}$, in the true vacuum state (TVS) $\chi \rightarrow \infty$. However, in the Einstein frame this singularity does not present and the energy density of TVS is zero without fine tuning of any scalar potential in $S_{1}$ or $S_{2}$ (see Ref. [9]). This means that even the weak version of the NGVE principle is enough to provide a solution of the CCP. We show in Subsec. IIIB how it is possible to incorporate gauge fields in such kind of model.

Sections IV and V are devoted to the ways for inclusion of fermions and construction of unified gauge theories in the context of the NGVE theory.

(C) As it is well known, in order to really understand the role of some symmetry, one should see what the breaking of such symmetry does. With a simple example in Sec.VI, we will see that the breaking of the symmetry (6) or (8) can lead to the appearance of a nonzero energy density for the TVS. In the particular example we study, the additional piece in the action that is added is of the form

$$
S_{3}=-\gamma \int \frac{\Phi^{2}}{\sqrt{-g}} d^{4} x .
$$

which is equivalent to considering of a piece of the Lagrangian density $L_{1}$ linear in $\chi$. Then as we show in Sec.VI, the TVS energy density appears to be equal to $\gamma$.

Notice that for the field theory models presented in this paper in the framework of the case (B) as well as of the case (C), the classical GR tests are guaranteed to work out correctly by the same resons as in the case (A) (provided that in the case (C), $\gamma$ in Eq. (9) is small enough).

In Sec.VII we show that when considering only a linear potential for a scalar field $\phi$ in $S_{1}$, the continuous symmetry $\phi \rightarrow \phi+$ const is respected. Surprisingly, in this case SSB takes place while no massless ("Goldstone") boson appears. Models with such a feature exist in each of the cases (A), (B), (C). 


\section{MODELS SATISFYING THE STRONG NGVE PRINCIPLE}

\section{A. General features of the strong NGVE theory}

Starting from the case (A) (see Introduction), we consider the action (5). We assume that the total Lagrangian density $L$ in Eq. (5) does not contain the measure fields $\varphi_{a}$, that is the fields by means of which the measure $\Phi$ is defined. If this condition is satisfied then the action (5) is invariant, up to a total divergence, under transformations (6) (see also [2]). In fact, by the transformation (6) the action (5) is changed according to $S \rightarrow S+\delta S$ with

$$
\delta S=4 \int A_{a}^{\mu} L \partial_{\mu} f_{a}(L) d^{4} x+6 \int \varepsilon^{\mu \nu \alpha \beta} \varepsilon_{a b c d} L \partial_{\mu} \varphi_{a} \partial_{\nu} \varphi_{b} \partial_{\alpha} L \partial_{\beta} L \frac{d f_{c}}{d L} \frac{d f_{d}}{d L}+\ldots
$$

where

$$
A_{a}^{\mu}=\varepsilon_{b c d a} \varepsilon^{\nu \lambda \sigma \mu}\left(\partial_{\nu} \varphi_{b}\right)\left(\partial_{\lambda} \varphi_{c}\right)\left(\partial_{\sigma} \varphi_{d}\right)
$$

and all terms in the right hand side of Eq. (10), with the exception of the first one, are identically equal to zero because of symmetry properties. The first term is transformed to the total divergence

$$
\delta S=\int \partial_{\mu} \Omega^{\mu} d^{4} x
$$

where $\Omega^{\mu} \equiv 4 A_{a}^{\mu} g_{a}(L)$ and $g_{a}(L)$ being defined from $f_{a}(L)$ through the equation $L\left(d f_{a} / d L\right)=d g_{a} / d L$ and the identity $\partial_{\mu} A_{a}^{\mu} \equiv 0$ has been taken into account.

Our choice for the total Lagrangian density is

$$
L=-\frac{1}{\kappa} R(\Gamma, g)+L_{m}
$$

where $L_{m}$ is the matter Lagrangian density and $R(\Gamma, G)$ is the scalar curvature which in the first order formalism in the framework of the Metric-Affine theory [10] is defined as follows

$$
\begin{gathered}
R(\Gamma, g)=g^{\mu \nu} R_{\mu \nu}(\Gamma) \\
R_{\mu \nu}(\Gamma)=R_{\mu \nu \lambda}^{\lambda}(\Gamma) \\
R_{\mu \nu \sigma}^{\lambda}(\Gamma) \equiv \Gamma_{\mu \nu, \sigma}^{\lambda}-\Gamma_{\mu \sigma, \nu}^{\lambda}+\Gamma_{\alpha \sigma}^{\lambda} \Gamma_{\mu \nu}^{\alpha}-\Gamma_{\alpha \nu}^{\lambda} \Gamma_{\mu \sigma}^{\alpha}
\end{gathered}
$$

where $\Gamma_{\mu \nu}^{\lambda}$ are the connection coefficients which have to be obtained from the variational principle.

Equations that originate from the variation of the action (5) with respect to the measure fields $\varphi_{a}$, are

$$
A_{a}^{\mu} \partial_{\mu}\left[-\frac{1}{\kappa} R(\Gamma, g)+L_{m}\right]=0
$$

Since $A_{a}^{\mu} \partial_{\mu} \varphi_{a^{\prime}}=4^{-1} \delta_{a a^{\prime}} \Phi$ we get 1 that $\operatorname{Det}\left(A_{a}^{\mu}\right)=\frac{4^{-4}}{4 !} \Phi^{3}$, so that if $\Phi \neq 0$, it follows from Eq. (17)

$$
-\frac{1}{\kappa} R(\Gamma, g)+L_{m}=M=\text { constant }
$$

Let us now study equations that originate from variation with respect to $g^{\mu \nu}$. For simplicity we present here the calculations for the case where there are no fermions. Performing the variation with respect to $g^{\mu \nu}$ we get

\footnotetext{
${ }^{1}$ For the connection with other developments concerning volume preserving diffeomorphisms and the corresponding structure of fields equations see Appendix A
} 


$$
-\frac{1}{\kappa} R_{\mu \nu}(\Gamma)+\frac{\partial L_{m}}{\partial g^{\mu \nu}}=0
$$

Contracting Eq.(19) with $g^{\mu \nu}$ and making use Eq.(18) we get

$$
g^{\mu \nu} \frac{\partial\left(L_{m}-M\right)}{\partial g^{\mu \nu}}-\left(L_{m}-M\right)=0
$$

This equation is a constraint since generically $L_{m}$ contains only the fields and their first derivatives. A similar constraint is achieved by using the vierbein - spin-connection (VSC) formalism (see Appendix E). Notice that if $L_{m}-M$ is homogeneous of degree one in $g^{\mu \nu}$ it satisfies the constraint (20) automatically (that is without using equations of motion).

\section{B. The vacuum case}

In the vacuum, when we choose $L_{m}=0$ in Eq.(13), it follows from Eq.(19)

$$
R_{\mu \nu}(\Gamma)=0
$$

Eq.(18) implies then that the integration constant $M=0$. Adding an arbitrary constant $C$ to $L_{m}$ does not change the resulting Eq.(21) since, as we see from Eqs. (18) and (19) or from the constraint (20), the integration constant $M$ has to compensate the constant $C$.

To clarify the sense of Eq. 211) we need the connection coefficients $\Gamma_{\mu \nu}^{\lambda}$. Varying the action (5) with $L=-\frac{1}{\kappa} R(\Gamma, g)$ with respect to $\Gamma_{\mu \nu}^{\lambda}$, we get

$$
\begin{aligned}
& -\Gamma_{\mu \nu}^{\lambda}-\Gamma_{\beta \mu}^{\alpha} g^{\beta \lambda} g_{\alpha \nu}+\delta_{\nu}^{\lambda} \Gamma_{\mu \alpha}^{\alpha}+\delta_{\mu}^{\lambda} g^{\alpha \beta} \Gamma_{\alpha \beta}^{\gamma} g_{\gamma \nu}- \\
& g_{\alpha \nu} \partial_{\mu} g^{\alpha \lambda}+\delta_{\mu}^{\lambda} g_{\alpha \nu} \partial_{\beta} g^{\alpha \beta}-\delta_{\nu}^{\lambda} \frac{\Phi, \mu}{\Phi}+\delta_{\mu}^{\lambda} \frac{\Phi,,_{\nu}}{\Phi}=0 .
\end{aligned}
$$

We will look for the solution of the form

$$
\Gamma_{\mu \nu}^{\lambda}=\left\{\begin{array}{l}
\lambda \\
\mu \nu
\end{array}\right\}+\Sigma_{\mu \nu}^{\lambda}
$$

where $\left\{\begin{array}{l}\lambda \\ \mu \nu\end{array}\right\}$ are the Christoffel's connection coefficients. Then $\Sigma_{\mu \nu}^{\lambda}$ satisfies the equation

$$
-\sigma,_{\lambda} g_{\mu \nu}+\sigma_{, \mu} g_{\nu \lambda}-g_{\nu \alpha} \Sigma_{\lambda \mu}^{\alpha}-g_{\mu \alpha} \Sigma_{\nu \lambda}^{\alpha}+g_{\mu \nu} \Sigma_{\lambda \alpha}^{\alpha}+g_{\nu \lambda} g_{\alpha \mu} g^{\beta \gamma} \Sigma_{\beta \gamma}^{\alpha}=0
$$

where

$$
\sigma \equiv \ln \chi, \quad \chi \equiv \frac{\Phi}{\sqrt{-g}}
$$

The general solution of Eq. (24) is

$$
\Sigma_{\mu \nu}^{\alpha}=\delta_{\mu}^{\alpha} \lambda, \nu_{\nu}+\frac{1}{2}\left(\sigma_{, \mu} \delta_{\nu}^{\alpha}-\sigma, \beta g_{\mu \nu} g^{\alpha \beta}\right)
$$

where $\lambda$ is an arbitrary function which appears due to the existence of the Einstein - Kaufman $\lambda$-symmetry (see [11], [1] and Appendix B): the curvature tensor (16) is invariant under the $\lambda$ - transformation

$$
\Gamma_{\mu \nu}^{\alpha \alpha}(\lambda, \sigma)=\Gamma_{\mu \nu}^{\alpha}+\delta_{\mu}^{\alpha} \lambda, \nu
$$

Although this symmetry was discussed in Ref. [11] in a very specific unified theory, it turns out that $\lambda$ - symmetry has a wider range of validity and in particular it is useful in our case.

If we choose the gauge $\lambda=\frac{\sigma}{2}$, then the antisymmetric part of $\Sigma_{\mu \nu}^{\alpha}$ disappears and we get

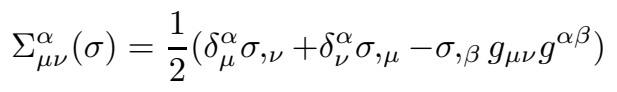


which contributes to the nonmetricity (more discussion about $\lambda$ symmetry and other gauge choices see Appendix B).

In the vacuum, the $\sigma$-contribution (28) to the nonmetricity can be eliminated. This is because in the vacuum $\left(L_{m}=0\right)$ the action (5), (13) is invariant under the local Einstein symmetry (LES)

$$
\begin{gathered}
g_{\mu \nu}(x)=J^{-1}(x) g_{\mu \nu}^{\prime}(x) \\
\Phi(x)=J^{-1}(x) \Phi^{\prime}(x)
\end{gathered}
$$

The transformation (30) can be the result of a diffeomorphism $\varphi_{a} \longrightarrow \varphi_{a}^{\prime}=\varphi_{a}^{\prime}\left(\varphi_{b}\right)$ in the space of the scalar fields $\varphi_{a}$ (see Ref. [3]). Then $J=\operatorname{Det}\left(\frac{\partial \varphi_{a}^{\prime}}{\partial \varphi_{b}}\right)$.

Notice that even when we are not in the vacuum, but the matter Lagrangian density $L_{m}$ satisfies the constraint (20) automatically (that is $L_{m}$ is homogeneous of degree one in $g^{\mu \nu}$, up to irrelevant additive constant) then the total action (5), (13) possesses LES too. For examples see [3], 代.

For $J=\chi$ we get $\chi^{\prime} \equiv 1, \quad \Sigma_{\mu \nu}^{\prime \alpha}(\sigma) \equiv 0$ and $\Gamma_{\mu \nu}^{\prime \alpha}=\left\{\begin{array}{c}\alpha \\ \mu \nu\end{array}\right\}^{\prime}$, where $\left\{\begin{array}{c}\alpha \\ \mu \nu\end{array}\right\}^{\prime}$ are the Christoffel's coefficients corresponding to the new metric $g_{\mu \nu}^{\prime}$. In terms of the new metric $g_{\mu \nu}^{\prime}$, the curvature (16) becomes the Riemannian curvature and therefore Eq. (21) is equivalent to the vacuum Einstein's equation with zero cosmological constant.

\section{Single scalar field with a nontrivial potential}

Now let us consider the cases when the constraint $(20)$ is not satisfied without restrictions on the dynamics of the matter fields. Nevertheless, the constraint (20) holds as a consequence of the variational principle in any situation.

A simple case where the constraint (20) is not automatic is the case of a single scalar field with a nontrivial potential $V(\varphi)$

$$
L_{m}=\frac{1}{2} \varphi_{, \alpha} \varphi^{, \alpha}-V(\varphi)
$$

In this model, the kinetic part of the action possesses LES and satisfies the constraint automatically since $\frac{1}{2} \varphi_{, \alpha} \varphi^{, \alpha}$ is homogeneous of degree one in $g^{\mu \nu}$. The potential part apparently does not satisfy the LES and as a result of this the constraint (20) implies

$$
V(\varphi)+M=0
$$

Therefore we conclude that, provided $\Phi \neq 0$, there is no dynamics for the theory of a single scalar field with a nontrivial potential, since constraint (32) forces this scalar field to be a constant.

The constraint (32) has to be solved together with the equation of motion resulting from the variation of the action (5), (13), (31) with respect to $\varphi_{a}$

$$
(-g)^{-1 / 2} \partial_{\mu}\left(\sqrt{-g} g^{\mu \nu} \partial_{\nu} \varphi\right)+\sigma,_{\mu} \varphi^{, \mu}+\frac{d V}{d \varphi}=0,
$$

where $\sigma=\ln \chi$. From eqs.(32) and (33) we conclude that the $\varphi$-field has to be located at an extremum of the potential $V(\varphi)$. Since the constraint(32) eliminates the dynamics of the scalar field $\varphi$, we cannot really say that we have a situation where the LES (29), (30) is actually broken, since after solving the constraint together with the equation of motion (i.e. on the mass shell) the symmetry remains true.

Taking into account that $\varphi=$ constant and $L_{m}-M=-(V(\varphi)+M)=0$, we see from Eqs. (18)-(20) that $R_{\mu \nu}(\Gamma, g)=0$. As we have seen in Sec. IIB, the $\sigma$ contribution to the connection can be eliminated in the vacuum by the transformations (29), (30). Notice that since the equations of motion enforce $\varphi=$ constant, the single scalar field $\phi$ part of the Lagrangian density acts as an arbitrary constant. However, this situation is indistinguishable from the vacuum case. Then repeating the LES transformation of the end of Sec. IIB, we see that in terms of the new metric $g_{\mu \nu}^{\prime}$, the tensor $R_{\mu \nu}(\Gamma, g)$ becomes the usual Ricci tensor $R_{\mu \nu}\left(g^{\prime}\right)$ of the Riemannian space-time with the metric $g_{\mu \nu}^{\prime}$ and $R_{\mu \nu}\left(g^{\prime}\right) \equiv 0$ Therefore we conclude that for the case of a single scalar field with a nontrivial potential, the theory is equivalent to the Einstein's GR with the zero cosmological constant. As a consequence, in this simple model, among maximally symmetric solutions, only Minkowski space is a solution. The absence of de Sitter space as a solution makes us suspect that the NGVE theory is inconsistent with the idea of inflation. This however is not true as we will see in the next subsection. 


\section{Four index field strength, unified gauge sector and a model with realistic particle fields dynamics and cosmology}

As we have mentioned, one of the biggest puzzles of modern physics is what is referred to as the "cosmological constant problem", i.e. the absence of a possible constant part of the vacuum energy in the present day universe [1]. On the other hand, many questions in modern cosmology appear to be solved by the so called "inflationary model" which makes use of a big effective cosmological constant in the early universe [12]. A possible conflict between a successful resolution of the cosmological constant problem and the existence of an inflationary phase could be a "potential Achilles heel for the scenario" as has been pointed out [13]. Here we will show (see also [5]) that indeed there is no conflict between the existence of an inflationary phase and the disappearance of the cosmological constant in the later phases of cosmological evolution (without the need of fine tuning). In the context of the strong NGVE theory, the introduction of a four index field strength condensate plays a crucial role for this.

Another problem related to the NGVE theory consists of the very strong restriction which constraint (20) dictates on the matter models which generally do not satisfy the LES. This makes the incorporation of fermion masses and gauge fields not straightforward (see Refs. [3], 目]). In what follows we will see, however, that the incorporation of the four index-field strength in four dimensional space - time turns the constraint into an equation for $\chi$ - field. After solving this constraint we obtain well defined matter models.

In Appendixes $\mathrm{C}$ and $\mathrm{D}$ we show how it is possible to realize a nontrivial dynamics of a scalar field and a gauge field while solving the CCP. However, the simplest models presented there give rise to undesirable problems: either the mass of the scalar field turns out to be infinite or nonminimal nonrenormalizable couplings appear at very high energies. We are going to show in this section that by a certain sort of unification of all gauge fields we can get rid of the above mentioned problem. This approach seems to be the most appealing one in the context of the theory based on the strong NGVE principle. This Section is self contained, but Appendixes C and D help the reader to understand how the theory operates generically.

The basic idea consists of demanding that the dependence of the Lagrangian density on the gradients of the gauge field potentials is only through a single variable which is the sum of all possible kinetic terms and a corresponding four index field strength term. The fact that all gauge fields must come together is automatic in a unified gauge models where the Lagrangian density must depend only on $F_{\mu \nu}^{a} F^{a \mu \nu}$ where $a$ is for example an $S U(5)$ index. In addition we insist also in introducing a three index potential $A_{\mu \nu \alpha}$ into the game in a similar way. Here we will demand that the field strength $F_{\alpha \mu \nu \beta}=\partial_{[\alpha} A_{\mu \nu \beta]}$ is combined with other usual gauge field kinetic terms, like $F_{\mu \nu}^{a} F^{a \mu \nu}$, in such a way that the homogeneity of each of the terms in $g^{\mu \nu}$ is of degree 2 . This singles out the following combination analytic in the gradients of the gauge fields potentials

$$
y \equiv F_{\mu \nu} F^{\mu \nu}+m^{2} \frac{\varepsilon^{\mu \nu \alpha \beta}}{\sqrt{-g}} \partial_{\mu} A_{\nu \alpha \beta}
$$

We will call $y$ the gauge complex. Here $m$ is a parameter with the dimensions of mass.

The demand that the term which depends on the condensate of $A_{\mu \nu \alpha}$, has to have the same transformation under $g^{\mu \nu} \rightarrow \Omega g^{\mu \nu}$ as the ordinary gauge fields, finds a simple analogy in a related higher dimensional picture where the components of the gauge field strengths in the direction of the extra dimensions can play a similar role to that of the $A_{\mu \nu \alpha}$ field in 4-dimensional space-time. In 6-dimensional case for example, $F_{A B} F^{A B} \equiv F_{\mu \nu} F^{\mu \nu}+2 F_{a \mu} F^{a \mu}+F_{a b} F^{a b}$, where $\mu, \nu=0,1,2,3 ; \quad a, b=4,5$ and $F_{a b} F^{a b}$ plays then the role of $\frac{\varepsilon^{\mu \nu \alpha \beta}}{\sqrt{-g}} \partial_{\mu} A_{\nu \alpha \beta}$ in the condensate state 16] (here $F_{a b}$ takes a "magnetic monopole" expectation value). Then the requirement of equal behavior under conformal transformation is of course automatic.

To implement the suggestion of using the combination (34) let us consider a model with the action

$$
S=\int \Phi d^{4} x\left[-\frac{1}{\kappa} R(\Gamma, g)-m^{4} f(u)+\frac{1}{2} g^{\mu \nu} \phi, \mu \phi,_{\nu}+g^{\mu \nu}\left(\partial_{\mu}-i \tilde{e} \tilde{A}_{\mu}\right) \phi\left(\partial_{\nu}+i \tilde{e} \tilde{A}_{\nu}\right) \phi^{*}-V(|\phi|)\right]
$$

where $\phi$ is a complex scalar field minimally coupled to a vector gauge field $\tilde{A}_{\mu}$ and $f(u)$ is a function of the dimensionless argument $u=y / m^{4}$. We will see that the only requirement condition on the function $f(u)$, that provides a persistent condensate with physically reasonable consequences, is that $f^{\prime}(u) \equiv \frac{d f}{d u}=0$ for some $y=y_{0}>0$.

\footnotetext{
${ }^{2}$ The other possibility including $\sqrt{-F_{\mu \nu \alpha \beta} F^{\mu \nu \alpha \beta}} \equiv\left|\frac{\varepsilon^{\mu \nu \alpha \beta}}{\sqrt{-g}} \partial_{\mu} A_{\nu \alpha \beta}\right|$ is not analytic one.
} 
By making use the gauge invariance we choose the unitary gauge (where $\operatorname{Im} \phi(x)=0$ ) and then the Lagrangian density takes the form

$$
S=\int \Phi d^{4} x\left[-\frac{1}{\kappa} R(\Gamma, g)-m^{4} f(u)+\frac{1}{2} g^{\mu \nu} \varphi_{\mu} \varphi,_{\nu}-V(\varphi)+\frac{1}{2} \tilde{e}^{2} \varphi^{2} g^{\mu \nu} \tilde{A}_{\mu} \tilde{A}_{\nu}\right]
$$

where $|\phi|=\frac{1}{2} \varphi$.

The constraint (20) has now the form

$$
-2 u f^{\prime}(u)+f(u)+\frac{1}{m^{4}}[V(\varphi)+M]=0 .
$$

Varying with respect to $A_{\nu \alpha \beta}$ we get

$$
\partial_{\mu}\left(\chi f^{\prime} \varepsilon^{\mu \nu \alpha \beta}\right)=0
$$

which gives

$$
\chi f^{\prime}=\omega
$$

where $\omega$ is a dimensionless integration constant.

Equation for the gauge field $\tilde{A}_{\mu}$

$$
\frac{1}{\sqrt{-g}} \partial_{\mu}\left[\chi f^{\prime} \sqrt{-g} \tilde{F}^{\mu \nu}\right]+\frac{\tilde{e}^{2}}{4} \varphi^{2} \chi \tilde{A}^{\nu}=0
$$

which is apparently nonlinear in $\tilde{F}^{\mu \nu}$ is reduced to the form

$$
\frac{1}{\sqrt{-g}} \partial_{\mu}\left[\sqrt{-g} \tilde{F}^{\mu \nu}\right]+\frac{\tilde{e}^{2}}{4 \omega} \varphi^{2} \chi g^{\nu \alpha} \tilde{A}_{\alpha}=0
$$

due to Eq. (39).

The gravitational equations originated by the variation of $g^{\mu \nu}$ take the form

$$
\frac{1}{\kappa} R_{\mu \nu}(\Gamma, g)=-\frac{1}{2} y f^{\prime} g_{\mu \nu}+\frac{1}{2} f^{\prime}\left[\tilde{F}^{\alpha \beta} \tilde{F}_{\alpha \beta} g_{\mu \nu}-4 \tilde{F}_{\mu \alpha} \tilde{F}_{\nu \beta} g^{\alpha \beta}\right]+\frac{1}{2} \varphi_{, \mu} \varphi_{, \nu}+\frac{\tilde{e}^{2}}{2} \varphi^{2} \tilde{A}_{\mu} \tilde{A}_{\nu}
$$

after using Eq. (34).

The scalar field equation is

$$
(-g)^{-1 / 2} \partial_{\mu}\left(\sqrt{-g} g^{\mu \nu} \partial_{\nu} \varphi\right)+\sigma_{, \mu} \varphi^{, \mu}+V^{\prime}(\varphi)+\tilde{e}^{2} \varphi g^{\alpha \beta} \tilde{A}_{\alpha} \tilde{A}_{\beta}=0,
$$

The $\chi$ - field enters both in the gravitational Eqs. (42) (through the connection) and in the matter equations (41), (43). In order to see easily the physical content of the model, we have to perform a conformal transformation

$$
\bar{g}_{\mu \nu}(x)=\chi g_{\mu \nu}(x) ; \quad \varphi \rightarrow \varphi
$$

to obtain an "Einstein picture". Notice that now this transformation is not a symmetry and indeed changes the form of equations. After rescaling $\tilde{A}_{\mu}$ and $\tilde{e}^{2}$ to $A_{\mu}$ and $e^{2}$

$$
A_{\mu} \equiv 2 \sqrt{\omega} \tilde{A}_{\mu} ; \quad e=\frac{\tilde{e}}{2 \sqrt{\omega}}
$$

with $\omega>0$, we obtain the resulting equations in the Einstein frame which are of canonical form for a standard SSB gauge theory in the GR formalism:

$$
\begin{gathered}
\frac{1}{\sqrt{-\bar{g}}} \partial_{\mu}\left(\sqrt{-\bar{g}} \bar{g}^{\mu \nu} \partial_{\nu} \varphi\right)+\frac{d V_{e f f}}{d \varphi}+e^{2} \varphi g^{\alpha \beta} A_{\alpha} A_{\beta}=0 \\
\frac{1}{\sqrt{-\bar{g}}} \partial_{\mu}\left(\sqrt{-\bar{g}} \bar{g}^{\mu \alpha} \bar{g}^{\nu \beta} F_{\alpha \beta}\right)+e^{2} \varphi^{2} \bar{g}^{\nu \alpha} A_{\alpha}=0
\end{gathered}
$$




$$
\begin{gathered}
G_{\mu \nu}\left(\bar{g}_{\alpha \beta}\right)=\frac{\kappa}{2} T_{\mu \nu} \\
T_{\mu \nu}=\varphi_{, \mu} \varphi_{, \nu}-\frac{1}{2} g_{\mu \nu} \varphi_{, \alpha} \varphi_{, \beta} \bar{g}^{\alpha \beta}+V_{e f f}(\varphi) \bar{g}_{\mu \nu}+\frac{1}{4} \bar{g}_{\mu \nu} F_{\alpha \beta} F^{\alpha \beta}-F_{\mu \alpha} F_{\nu \beta} \bar{g}^{\alpha \beta}+e^{2} \varphi^{2}\left(A_{\mu} A_{\nu}-\frac{1}{2} \bar{g}_{\mu \nu} A_{\alpha} A^{\alpha}\right)
\end{gathered}
$$

Here

$$
G_{\mu \nu}\left(\bar{g}_{\alpha \beta}\right) \equiv R_{\mu \nu}\left(\bar{g}_{\alpha \beta}\right)-\frac{1}{2} \bar{g}_{\mu \nu} R\left(\bar{g}_{\alpha \beta}\right)
$$

is the Einstein tensor in the Riemannian space-time with metric $\bar{g}_{\mu \nu}$.

The new effective scalar field potential appears now in two different forms in Eqs. (46) and (49):

$$
\frac{d V_{\text {eff }}}{d \varphi} \equiv V_{e f f}^{\prime}=\frac{1}{\chi} \frac{d V}{d \varphi}=\frac{1}{\omega} \frac{d f}{d u} \frac{d V}{d \varphi}
$$

and

$$
V_{e f f}(\varphi)=\frac{y}{\omega}\left(f^{\prime}(u)\right)^{2}
$$

Here $y, u \equiv y / m^{4}$ and $f(u)$ are functions of $\varphi$ due to the constraint (37). It can be shown that two different form of appearance of $V_{\text {eff }}$ and $V_{\text {eff }}^{\prime}$ in the gravitational field equations and in the scalar field equation correspondingly, are self consistent (the reason for this is the existence of Bianchi identities). This consistency also may be shown by taking the derivative of Eq. (52) with respect to $\varphi$ and using the derivative of the constraint (37) with respect to $\varphi$. As the result we obtain Eq. (51).

Looking at Eq. (51) we see that there are two ways of obtaining an extremum of $V_{\text {eff }}(\varphi)$ :

(a) The first one is when $\frac{d V}{d \varphi}=0$ which corresponds to an extremum of the original potential $V(\varphi)$. In this case there is no reason for the vanishing $V_{e f f}(\varphi)$ in such an extremum if we do not resort to some kind unnatural fine tuning.

(b) The second way is to consider the situation where

$$
\left.\frac{d f}{d u}\right|_{u=u_{0} \neq 0}=0
$$

and the appropriate value of $\varphi_{0}$ is related to $u_{0}$ by the constraint (37). For this extremum of the effective potential we see immediately from Eq. (52) that $V_{e f f}\left(\varphi_{0}\right)=0$ without any assumption about $\frac{d V}{d \varphi}$, that is without fine tuning.

If we assume that $y_{0}=\frac{u_{0}}{m^{4}}$ is positive then it is clear also from (52) that $\varphi_{0}$ (where both $V_{\text {eff }}\left(\varphi_{0}\right)=0$ and $\left.\left.\frac{d V_{e f f}}{d \varphi}\right|_{\varphi=\varphi_{0}}=0\right)$, is a minimum since any small fluctuations bring us to a higher positive value of $V_{\text {eff }}$. In this case the vacuum is defined both by value of the gauge complex condensate $u=u_{0}$ and by the scalar condensate $\varphi=\varphi_{0}$ satisfying the condition

$$
f\left(u_{0}\right)+\frac{1}{m^{4}}\left[V\left(\varphi_{0}\right)+M\right]=0 .
$$

which follows from the constraint (37) and Eq.(53). Notice that transition to the Einstein frame does not change the value of the gauge complex condensate $u_{0}$ since it is defined by the value of $\varphi_{0}$ due to Eq. (54).

It is very important to notice that Eq. (54) represents the exact mutual cancellation of the contributions to the vacuum energy of the integration constant $M$, the scalar field condensate and the gauge complex condensate.

It can be shown explicitly (by using the constraint (37), its derivative and Eq. (51) that the mass square of the scalar particle is

$$
V_{e f f}^{\prime \prime}\left(\varphi_{0}\right)=\frac{1}{2 \omega y_{0}}\left[V^{\prime}\left(\varphi_{0}\right)\right]^{2} .
$$

which is positive if both $\omega>0$ and $y_{0}>0$. In this case the state $y=y_{0}, y=y_{0}$ is classically stable and it has zero energy density. In this state, the vector boson $A_{\mu}$ acquires mass

$$
m_{A}^{2}=e^{2} \varphi_{0}^{2}
$$


As it has been noted in Introduction, equations of motion derived from the action (5) are invariant under the global dilatation of the measure fields $\varphi_{a}$ :

$$
\varphi_{a} \rightarrow \alpha_{a} \varphi_{a}, \quad \alpha_{a}=\text { const }
$$

or in terms of the measure $\Phi$

$$
\Phi \rightarrow \alpha \Phi, \quad \alpha=\prod \alpha_{a}
$$

In the Einstein frame (see Eq. (44)) this symmetry takes the form of a global space-time dilatation

$$
\Phi(x) \rightarrow \alpha \Phi(x) ; \quad \bar{g}_{\mu \nu}(x) \rightarrow \alpha \bar{g}_{\mu \nu}(x) ; \quad(\chi(x) \rightarrow \alpha \chi(x))
$$

The appearance of the gauge complex condensate (39) parameterized by the integration constant $\omega$, spontaneously breaks this symmetry. As we can see from Eqs. (45), (56) and (55) all coupling constants and masses are defined by the dilatation breaking integration constant $\omega$.

One can see that if $y>0$ in the extremum where $\frac{d V}{d \varphi}=0$, then this state can serve as a phase with positive effective cosmological constant and therefore inflation becomes possible as well. This vacuum is smoothly connected by dynamical evolution of the scalar field $\varphi$ with the zero energy density vacuum $y=y_{0}, y=y_{0}$, thus providing a way to achieve inflation and transition (after a standard reheating period) to a $\Lambda=0$ phase without fine tuning.

\section{MODELS SATISFYING THE WEAK NGVE PRINCIPLE}

\section{A. General features of the weak NGVE theory}

As we have seen in Introduction and in Sec. IIA, an action of the form (5) is invariant (up to an integral of a total divergence) under the SGMF transformations (6). However, the existence of such symmetry is not affected by the existence of an additional contribution to the action of the form $S_{2}$ in Eq. (7). In this case the SGMF symmetry is modified as in Eq. (8). In this section we want to study this more general structure. Notice that in the weak NGVE theory, the invariance of classical equations of motion under the global dilaton transformations (58) is explicitly violated.

We are starting now from Eq. (7) in the case when neither $L_{1}$ nor $L_{2}$ contain the measure fields $\varphi_{a}$ and where for simplicity we assume that no $\Gamma_{\mu \nu}^{\lambda}$ - dependence enters in $L_{2} \equiv L_{m 2}=L_{m 2}\left(g_{\mu \nu}\right.$, matterfields $)$ and $L_{1}$ has the form $L_{1}=-\frac{1}{\kappa} R(g, \Gamma)+L_{m 1}\left(g_{\mu \nu}\right.$, matter fields $)$.

Variation with respect to the measure fields $\varphi_{a}$ leads to the equation

$$
A_{a}^{\mu} \partial_{\mu}\left[-\frac{1}{\kappa} R(\Gamma, g)+L_{m 1}\right]=0
$$

Just as in Sec. IIA, if $\Phi \neq 0$, it follows from Eq. (60)

$$
-\frac{1}{\kappa} R(\Gamma, g)+L_{m 1}=M=\text { constant }
$$

Varying with respect to $g^{\mu \nu}$ we get

$$
\Phi\left(-\frac{1}{\kappa} R_{\mu \nu}(\Gamma)+\frac{\partial L_{m 1}}{\partial g^{\mu \nu}}\right)-\frac{1}{2} \sqrt{-g} L_{m 2} g_{\mu \nu}+\sqrt{-g} \frac{\partial L_{m 2}}{\partial g^{\mu \nu}}=0
$$

The consistency condition of Eqs. (61) and (62) is the constraint

$$
g^{\mu \nu}\left(\frac{\partial L_{m 1}}{\partial g^{\mu \nu}}+\frac{1}{\chi} \frac{\partial L_{m 2}}{\partial g^{\mu \nu}}\right)-\left(L_{m 1}+\frac{2}{\chi} L_{m 2}-M\right)=0
$$

which takes place of the constraint (20) of Sec. IIA. Notice that constraint (63) is satisfied automatically (that is without using equations of motions) in the case when $L_{m 1}$ and $L_{m 2}$ are homogeneous functions of $g^{\mu \nu}$ of degree 1 and 2 correspondingly. In such a case the theory possesses the LES (29), (30) and then, similarly to the strong NGVE theory (see the end of Sec.IIB), the $\chi$ contributions into equations of motion can be eliminated by the transformation (29), (30) with $J=\chi$. 


\section{B. The simplest model}

To demonstrate how the theory works when it is based on the weak NGVE principle, we start here from the simplest model [8], [9] including scalar field $\varphi$ and gravity according to the prescription of the NGVE principle and in addition to this we include the standard cosmological constant term. So, we consider an action

$$
S=\int L_{1} \Phi d^{4} x+\int \Lambda \sqrt{-g} d^{4} x
$$

where $L_{1}=-\frac{1}{\kappa} R(\Gamma, g)+\frac{1}{2} g^{\mu \nu} \varphi_{, \mu} \varphi_{, \nu}-V(\varphi)$ and $\Lambda=$ const.. Notice that now the global dilatation symmetry (58) is explicitly broken.

Performing the variation with respect to the measure fields $\varphi_{a}$ we obtain equations $A_{a}^{\mu} \partial_{\mu} L_{1}=0$ where $A_{b}^{\mu}$ is given by Eq. (11). If $\Phi \neq 0$, then it follows from the last equations that $L_{1}=M=$ const.

Varying the action (64) with respect to $g^{\mu \nu}$ we get

$$
\Phi\left(-\frac{1}{\kappa} R_{\mu \nu}(\Gamma)+\frac{1}{2} \varphi_{, \mu} \varphi_{, \nu}\right)-\frac{1}{2} \sqrt{-g} \Lambda g_{\mu \nu}=0
$$

Contracting Eq.(65) with $g^{\mu \nu}$ and using equation $L_{1}=M$ we obtain the constraint

$$
M+V(\varphi)-\frac{2 \Lambda}{\chi}=0
$$

where again $\chi \equiv \Phi / \sqrt{-g}$.

The scalar field $\varphi$ equation has the same form as Eq. (33).

The derivatives of the field $\sigma$ enter both the gravitational equation (65) (through the connection) and in the scalar field equation. By a conformal transformation (44) to an "Einstein picture" we get, after using the constraint (66), the canonical form of equation for the scalar field and of the gravitational equations in the Riemannian space-time with metric $\bar{g}_{\alpha \beta}$

$$
\begin{aligned}
& \frac{1}{\sqrt{-\bar{g}}} \partial_{\mu}\left(\sqrt{-\bar{g}} \bar{g}^{\mu \nu} \partial_{\nu} \varphi\right)+\frac{d V_{\text {eff }}}{d \varphi}=0 \\
& R_{\mu \nu}\left(\bar{g}_{\alpha \beta}\right)-\frac{1}{2} \bar{g}_{\mu \nu} R\left(\bar{g}_{\alpha \beta}\right)=\frac{\kappa}{2} T_{\mu \nu}^{e f f}(\varphi)
\end{aligned}
$$

where

$$
T_{\mu \nu}^{e f f}(\varphi)=\varphi_{, \mu} \varphi_{, \nu}-\frac{1}{2} \bar{g}_{\mu \nu} \varphi_{, \alpha} \varphi_{, \beta} \bar{g}^{\alpha \beta}+\bar{g}_{\mu \nu} V_{e f f}(\varphi)
$$

and

$$
\begin{aligned}
\frac{d V_{e f f}}{d \varphi} & =\frac{1}{\chi} \frac{d V}{d \varphi} \\
V_{e f f}(\varphi) & =\frac{1}{4 \Lambda}[M+V(\varphi)]^{2}
\end{aligned}
$$

We see that for any analytic function $V(\varphi)$, the effective potential in the Einstein picture has an extremum, i.e. $V_{e f f}^{\prime}=0$, either when $V^{\prime}=0$ or $V+M=0$. The extremum $\varphi=\varphi_{1}$ where $V^{\prime}\left(\varphi_{1}\right)=0$ has nonzero energy density $\left[M+V\left(\varphi_{1}\right)\right]^{2} / 4 \Lambda$ if a fine tuning is not assumed. In contrast to this, if $\Lambda>0$, the state $\varphi=\varphi_{0}$ where $V\left(\varphi_{0}\right)+M=0$ is the absolute minimum and therefore $\varphi_{0}$ is a true vacuum with zero cosmological constant without any fine tuning. A mass square of the scalar field describing small fluctuations around $\varphi_{0}$ is $m^{2}=\frac{1}{2 \Lambda}\left[V^{\prime}\left(\varphi_{0}\right)\right]^{2}$. Exploiting the possibility to choose any analytic $V(\varphi)$, we can pick the structure of $V_{\text {eff }}(\varphi)$ so that it allows for an inflationary era, the possibility of reheating after scalar field oscillations and the setting down to a zero cosmological constant phase at the later stages of cosmological evolution, without fine tuning.

Notice that if $V+M$ reaches the value zero at some value of $\varphi=\varphi_{0}$, this point represents the absolute minimum of the effective potential. If this is not the case for a particular choice of potential $V$ and of integration constant $M$, it is always possible to choose an infinite range of values of $M$ where this will happen. Therefore no fine tuning of parameters has to be invoked, the zero value of the true vacuum energy density appears naturally in this theory. 
It is interesting that in the TVS $\chi \rightarrow \infty$, that is the dynamical measure $\Phi$ dominates the measure $\sqrt{-g}$. Therefore, in the TVS we observe a dominance of the first term (even if $\Lambda$ is very big) in Eq. (64) which implies the restoration of the global dilatation symmetry (58) in the TVS.

As an example let us consider the model with the quadratic potential $V(\varphi)=\frac{\mu^{2}}{2} \varphi^{2}$, which in the standard GR is associated with massive non selfinteracting real scalar field theory. In the theory under consideration, however, we obtain a very different result: the effective potential in the Einstein picture appears

$$
V_{\text {eff }}=\frac{\mu^{4}}{16 \Lambda}\left(\varphi^{2}-\varphi_{0}^{2}\right)^{2}, \quad \varphi_{0}^{2}=-\frac{2 M}{\mu^{2}}
$$

Notice that it follows from the constraint (66) that $M=\frac{2 \Lambda}{\chi}-\frac{1}{2} \mu^{2} \varphi^{2} \rightarrow-\frac{1}{2} \mu^{2} \varphi^{2}$ as $\varphi$ approaches the TVS (that is $\left.\chi^{-1} \rightarrow 0\right)$. Therefore, if $\mu^{2}>0$, then for the TVS to exist, the integration constant $M$ must be $M \leq 0$.

We see that for $\Lambda>0$, spontaneous breaking of the discrete symmetry $\varphi \rightarrow-\varphi$ takes place if $M \mu^{2}<0$. Yet, the vacuum energy at the absolute minimum $\varphi= \pm\left|\varphi_{0}\right|$ is zero without fine tuning. Furthermore, the mass of the scalar field is $m^{2}=\frac{\mu^{4}}{2 \Lambda} \varphi_{0}^{2}=-\frac{M \mu^{2}}{\Lambda}$ which as we see depends on the integration constant $M$. The mass $m$ is therefore a "floating" physical parameter, since $M$ does not appear in the original Lagrangian but it is determined by initial conditions of the Universe.

If $\varphi$ is replaced by a complex field $\phi$ and $\varphi^{2}$ by $\phi^{*} \phi$, we obtain the SSB of a continuous symmetry with standard consequences. In addition, a model of cosmology that can include an inflationary phase taking place in a false vacuum and transition to a zero cosmological constant phase is obtained without fine tuning.

Further generalizations do not modify the qualitative nature of the effects described here and they will be studied elsewhere. For example, considering a term of the form $\int U(\varphi) \sqrt{-g} d^{4} x$ instead of $\Lambda \int \sqrt{-g} d^{4} x$ in Eq. 64), one can see that the resulting effective potential vanishes when $V\left(\varphi_{0}\right)+M=0$ if $U\left(\varphi_{0}\right) \neq 0$ since it equals $V_{\text {eff }}=\frac{1}{4 U(\varphi)}(V+M)^{2}$.

A model based on the weak NGVE principle which incorporates global scale invariance is considered in Refs. 14]. and 115$]$.

\section{Including the gauge fields}

The weak NGVE principle allows to incorporate gauge fields in a way which is simpler than in the context of the strong NGVE principle. Taking in Eqs. (7)

$$
\begin{aligned}
L_{1} & =-\frac{1}{\kappa} R(\Gamma, g)+g^{\mu \nu}\left(\partial_{\mu}-i e A_{\mu}\right) \phi\left(\partial_{\nu}+i e A_{\nu}\right) \phi^{*}-V(\phi) \\
L_{2} & =-\frac{1}{4} g^{\alpha \beta} g^{\mu \nu} F_{\alpha \mu} F_{\beta \nu}+\Lambda
\end{aligned}
$$

we see that except of the $V$-term in $L_{1}$ and $\Lambda$-term in $L_{2}$, the action (7) is invariant under the LES (notice that in $S_{2}$ the LES takes the form of the conformal transformation of the metric $g_{\mu \nu}(x)=J^{-1}(x) g_{\mu \nu}^{\prime}(x)$ ). Therefore, as one can check explicitly, the gauge field does not enter in the constraint which turns out to be identical to Eq. (66).

For the gauge field in the unitary gauge we get

$$
(-g)^{-1 / 2} \partial_{\mu}\left(\sqrt{-g} g^{\mu \alpha} g^{\nu \beta} F_{\alpha \beta}\right)+e^{2} \chi \varphi^{2} g^{\mu \nu} A_{\mu}=0
$$

In the Einstein conformal frame where the metric is $\bar{g}_{\mu \nu}=\chi g_{\mu \nu}$, the gauge field equation takes the canonical form

$$
\frac{1}{\sqrt{-\bar{g}}} \partial_{\mu}\left(\sqrt{-\bar{g}} \bar{g}^{\mu \alpha} \bar{g}^{\nu \beta} F_{\alpha \beta}\right)+e^{2} \varphi^{2} \bar{g}^{\mu \nu} A_{\mu}=0
$$

The gravitational and scalar field equations including the effective scalar field potential in the Einstein picture coincide with the appropriate equations of the model in the previous Subsection IIIB. Keeping the assumption that $\Lambda>0$ we can see that all conclusions concerning the vanishing of the vacuum energy in the TVS $\varphi=\varphi_{0}$ where $V\left(\varphi_{0}\right)+M=0$ remain unchanged. In addition to this, the Higgs mechanism for the mass generation in the true vacuum state $\varphi_{0}$ works now in a regular way in contrast to the model based on the strong NGVE principle where the effective coupling constant (and as a consequence, the mass of the gauge boson) depends on the integration constant. 


\section{THE INCLUSION OF FERMIONS}

\section{A. Fermions in the NGVE theory}

To present a complete enough picture let us consider a strong NGVE model including gravity, gauge and scalar field sectors (as in the action (36), once again in the unitary gauge) and, in addition, the fermionic sector (for notations see Appendixes E and F):

$$
\begin{aligned}
& S=\int \Phi d^{4} x\left[-\frac{1}{\kappa} V^{a \mu} V^{b \nu} R_{\mu \nu a b}(\omega)-m^{4} f(u)+\frac{1}{2} g^{\mu \nu} \varphi, \mu \varphi, \nu-V(\varphi)+\frac{1}{2} \tilde{e}^{2} \varphi^{2} g^{\mu \nu} \tilde{A}_{\mu} \tilde{A}_{\nu}\right. \\
& \left.+\frac{i}{2} \bar{\Psi}\left\{\gamma^{a} V_{a}^{\mu}\left(\vec{\partial}_{\mu}+\frac{1}{2} \omega_{\mu}^{c d} \sigma_{c d}-i \tilde{e} \tilde{A}_{\mu}\right)-\left(\overleftarrow{\partial}_{\mu}-\frac{1}{2} \omega_{\mu}^{c d} \sigma_{c d}+i \tilde{e} \tilde{A}_{\mu}\right) \gamma^{a} V_{a}^{\mu}\right\} \Psi+U(\bar{\Psi} \Psi)\right]
\end{aligned}
$$

where the selfinteraction term $U(\bar{\Psi} \Psi)$ depending on the argument $\bar{\Psi} \Psi$ remains unspecified in this subsection.

Equation for $\Psi$ which follows from the action (76) is

$$
\left\{i\left[V_{a}^{\mu} \gamma^{a}\left(\partial_{\mu}-i \tilde{e} \tilde{A}_{\mu}\right)+\gamma^{a} C_{a b}^{b}+\frac{1}{4} \omega_{\mu}^{c d}\left(\gamma^{a} \sigma_{c d}+\sigma_{c d} \gamma^{a}\right) V_{a}^{\mu}\right]+\frac{1}{2} \gamma^{a} V_{a}^{\mu} \sigma_{, \mu}+U^{\prime}(\bar{\Psi} \Psi)\right\} \Psi=0
$$

where

$$
C_{a b}^{b}=\frac{1}{2 \sqrt{-g}} \partial_{\mu}\left(\sqrt{-g} V_{a}^{\mu}\right)
$$

is the trace of the so-called Ricci rotation coefficients [17] and $U^{\prime}$ is derivative of $U$ with respect to its argument $\bar{\Psi} \Psi$. Spin-connection $\omega_{\mu}^{c d}$ is defined by Eqs. (F4)- (E7). Remind that $\sigma$-field is defined by Eq. (25).

After the transition to the Einstein frame by means of the conformal transformations

$$
\begin{gathered}
V_{a \mu}^{\prime}(x)=\chi^{1 / 2}(x) V_{a \mu}(x) \\
\Psi^{\prime}(x)=\chi^{-1 / 4}(x) \Psi(x) ; \bar{\Psi}^{\prime}(x)=\chi^{-1 / 4}(x) \bar{\Psi}(x) \\
\varphi \rightarrow \varphi ; \tilde{A}_{\mu} \rightarrow \tilde{A}_{\mu}
\end{gathered}
$$

and performing the rescalings 445$)$ Eq. (77) is reduced to the form

$$
\left\{i\left[V_{a}^{\prime \mu} \gamma^{a}\left(\partial_{\mu}-i e A_{\mu}\right)+\gamma^{a} C_{a b}^{\prime b}+\frac{i}{4} \omega_{\mu}^{\prime c d} \varepsilon_{a b c d} \gamma^{5} \gamma^{b} V^{a \mu}\right]+\chi^{-1 / 2} U^{\prime}\left(\bar{\Psi}^{\prime} \Psi^{\prime}\right)\right\} \Psi^{\prime}=0
$$

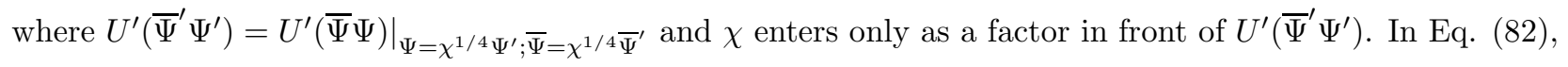

$$
C_{a b}^{\prime b}=\frac{1}{2 \sqrt{-g^{\prime}}} \partial_{\mu}\left(\sqrt{-g^{\prime}} V_{a}^{\prime \mu}\right)
$$

and the spin-connection $\omega_{\mu}^{\prime c d}$ is

$$
\omega_{\mu}^{\prime c d}=\omega_{\mu}^{c d}\left(V_{a}^{\prime \alpha}\right)+K_{\mu}^{c d}\left(V_{a}^{\prime \alpha}, \Psi^{\prime}, \bar{\Psi}^{\prime}\right)
$$

Notice that after the conformal transformation (79)-(81), the $\sigma$-contribution to the spin-connection (the second term in the r.h.s. of Eq. (F4)) is canceled.

Notice also that once again we performed the rescaling (45) in order to provide the standard form of the appropriate equations for the gauge and gravitational fields. 


\section{B. Nambu - Jona-Lasinio model in the NGVE theory}

As it is shown in Appendix E, the famous Nambu - Jona-Lasinio (NJL) model [18] works in a special way in the context of the NGVE theory: in the theory with only fermionic matter, the constraint does not impose restrictions on dynamics (see also [3]).

If we generalize the model towards a realistic theory by the consideration both bosonic and fermionic sectors as in the previous subsection, then still the fermionic part does not contribute to the constraint if we restrict ourselves to the NJL - type model. In this case the constraint works exactly as in Sec. III where bosonic sector breaks the LES explicitly and the constraint becomes an equation for $\chi$. The modification of the NJL type model for the case of the weak NGVE theory (see Eq. (7)) is evident if the fermion sector enter only in $S_{1}$.

In the Einstein frame, the NJL term (see the last term in Eq. (82)) remains its original form in terms of the transformed fields $\Psi^{\prime}$ and $\bar{\Psi}^{\prime}$.

As it is well known 19], the the NJL mechanism allows for a dynamical mass generation in realistic models. Since in the Einstein frame the theory takes the canonical form, therefore the same mechanisms of the dynamical mass generation can be applied also here.

\section{Classical model for generating mass of fermions}

As we have discussed in the previous subsection, there is a possibility to generate masses of fermions in the NGVE theory due to the quantum effect in the NJL model. Now we are going to show that the NGVE theory in the context of the model with the action (76) allows for a purely classical mechanism of obtaining fermion masses.

The constraint (E7) corresponding to the model (76) is

$$
\bar{\Psi} \Psi U^{\prime}(\bar{\Psi} \Psi)-2 U(\bar{\Psi} \Psi)-2\left[m^{4}\left(2 u f^{\prime}(u)-f(u)\right)-(V(\varphi)+M)\right]=0
$$

where Eq. (E10) has been used.

Directing our attention to the situation where the scalar field excitations around the vacuum $\left\{u=u_{0}, \varphi=\varphi_{0}\right.$ with condition (37) $\}$, (see Sec.IID) are ignored, we get the constraint for the first order fluctuations of $\Psi$ and $\bar{u} \equiv u-u_{0}$

$$
\bar{\Psi} \Psi U^{\prime}(\bar{\Psi} \Psi)-2 U(\bar{\Psi} \Psi)-4 m^{4} u_{0} f^{\prime \prime}\left(u_{0}\right) \bar{u}=0
$$

where Eq. (53) has been used.

We consider now the model with fermionic selfinteraction of the form

$$
U(\bar{\Psi} \Psi)=-C(\bar{\Psi} \Psi)^{q}, \quad C=k m^{4-3 q}
$$

where $k>0$ is a dimensionless parameter.

Remembering that our aim is to study the theory in the Einstein frame, we first calculate $\chi$. From Eq. (39) we have $\chi=\frac{\omega}{f^{\prime}}$ and expanding $f^{\prime}$ around $u=u_{0}$ we get $f^{\prime}=f^{\prime}\left(u_{0}\right)+f^{\prime \prime}\left(u_{0}\right) \bar{u}+\ldots=f^{\prime \prime}\left(u_{0}\right) \bar{u} . \bar{u}$ can be obtained from Eq. (86) and for special form (87) we obtain, after a simple computation, the following equation for $\chi$

$$
\frac{\omega}{\chi}=\frac{C}{4 u_{0} m^{4}}\left[(2-q) \chi^{q / 2}\left(\bar{\Psi}^{\prime} \Psi^{\prime}\right)^{q}\right]
$$

in terms of the fermion field $\Psi^{\prime}$ in the Einstein frame (see Eq. (80)), that is

$$
\chi=\left(\frac{C(2-q)}{4 \omega u_{0} m^{4}}\right)^{-\frac{2}{q+2}}\left(\bar{\Psi}^{\prime} \Psi^{\prime}\right)^{-\frac{2 q}{q+2}}
$$

Finally, we observe that in the Einstein frame, the last term in Eq. (82) has the form of a mass term, with a mass $m_{f}$ given by

$$
m_{f}=C q \chi^{-1 / 2}(\bar{\Psi} \Psi)^{q-1}=C q \chi^{\frac{q-2}{2}}\left(\bar{\Psi}^{\prime} \Psi^{\prime}\right)^{q-1}
$$


Such a term will be a legitimate mass term only if $m_{f}$ as given by Eq. (90) is a genuine constant. From Eqs. (89) and (90) we obtain that $m_{f}$ is given by

$$
m_{f}=C q\left(\frac{C(2-q)}{4 \omega u_{0} m^{4}}\right)^{\frac{2-q}{q+2}}\left(\bar{\Psi}^{\prime} \Psi^{\prime}\right)^{\frac{3 q-2}{q+2}}
$$

We observe therefore that $m_{f}$ is indeed a genuine constant if $q=2 / 3$ :

$$
m_{f}=\frac{m k^{3 / 2}}{\sqrt{3 \omega u_{0}}}
$$

We conclude therefore that if we start with a fermion selfinteraction term in the original Lagrangian density (see Eq. $(76))$ of the form

$$
U(\bar{\Psi} \Psi)=-C(\bar{\Psi} \Psi)^{2 / 3},
$$

we obtain normal propagation of a massive fermion in the (physical) Einstein frame.

If we had taken the normal fermion mass term in the original frame, that is $q=1$, then in the absence of fermionic condensate we would get zero fermion mass in the Einstein frame.

One should notice at this point that a selfinteraction of the form (93) has a remarkable feature: such a term comes in the action with a coupling constant $C=\mathrm{km}^{2}$ with dimensionality $\mathrm{mass}^{2}$, just as we are used in the case of bosonic masses, like in the case of a vector meson mass term for example.

\section{UNIFIED GAUGE THEORIES IN THE CONTEXT OF NGVE THEORY}

Now we able to formulate a realistic gauge theory. For the illustration of this we take $S U(2) \times U(1)$ model of electroweak interaction. However, there are no obstacles to formulate QCD, GUT or any other spontaneously broken gauge model in the context of the NGVE theory. The common feature of such models in the NGVE theory is that the spontaneous symmetry breaking (SSB) in the vacuum $\left\{\varphi_{0}, y_{0}\right\}$ does not generate the vacuum energy and therefore the cosmological constant is equal to zero in this vacuum. We present here the model realized in the context of the strong NGVE theory with the use of the 4-index field strength. However, unified gauge theories can be formulated without the 4-index field strength following the guide-line of the Sec. III, that is in the context of the weak NGVE theory.

The $S U(2) \times U(1)$ model of electroweak interaction in the NGVE theory has to be considered together with gravitational interaction and the Lagrangian density is the following

$$
\begin{array}{r}
L=-\frac{1}{\kappa} V^{a \mu} V^{b \nu} R_{\mu \nu a b}(\omega)-m^{4} f(u)+i \bar{L} \not D L+i \bar{e}_{R} \not D e_{R}+i \bar{\nu}_{R} \not D \nu_{R} \\
+\left|D_{\mu} \varphi\right|^{2}-V(|\varphi|)-\lambda_{e} m^{4 / 3}\left(\bar{L} e_{R} \varphi+\text { h.c. }\right)^{2 / 3}
\end{array}
$$

where we choosed the classical model for generating mass of fermions (see Sec. IVC).

In Eq. (94) we used notations of Ref. [20]: $S U(2)$ vector gauge field $\tilde{A}_{i \mu},(i=1,2,3.) ; \quad U(1)$ abelian gauge field $\tilde{B}_{\mu} ; \quad$ Left lepton dublet

$$
L=\left(\begin{array}{c}
\nu_{L} \\
e_{L}
\end{array}\right)
$$

Right $S U(2)$ singlets $\nu_{R}$ and $e_{R} ; S U(2)$ scalar fields dublet

$$
\varphi=\left(\begin{array}{c}
\varphi^{+} \\
\varphi^{0}
\end{array}\right)
$$

and corresponding antiparticles $\varphi^{\dagger}=\left(\varphi^{-}, \tilde{\varphi}^{0}\right)$. The left and right components of fermions are defined by $\Psi_{L} \equiv$ $\frac{1}{2}\left(1+\gamma_{5}\right) \Psi$ and $\Psi_{L} \equiv \frac{1}{2}\left(1-\gamma_{5}\right) \Psi$ correspondingly; $D_{\mu}=\partial_{\mu}-i g T_{i} \tilde{A}_{i \mu}-i g^{\prime} \frac{Y}{2} \tilde{B}_{\mu}$ where the hypercharge $Y$ is: $Y=-1$ for $\nu_{L}$ and $e_{L} ; \quad Y=-2$ for $e_{R} . \quad T_{i}=\frac{1}{2} \tau_{i}$ for $\quad \varphi$ and $L$. For isoscalar fields $e_{R}$ and $\nu_{R}, \quad T=0$. The last 
term in (94) is written in the form which provides us the mechanism for the fermion mass generation described in Sec.IVC and accompanied by SSB. Parameter $\lambda_{e}$ in the last term of Eq. (94) is dimensionless coupling constant.

Operator $\not D$ in the third term $\bar{L} \not D L$ of Eq. (94) is defined as follows:

$$
\not D \equiv \overrightarrow{D D}_{L}-\overleftarrow{\not D}_{L}
$$

where

$$
\begin{gathered}
\overrightarrow{D D}_{L} \equiv \frac{1}{2} V_{a}^{\mu} \gamma^{a}\left(\vec{\partial}_{\mu}+\frac{1}{2} \omega_{\mu}^{c d} I \sigma_{c d}-\frac{i}{2} \tilde{g} \tau_{i} \tilde{A}_{i \mu}+\frac{i}{2} \tilde{g}^{\prime} \tilde{B}_{\mu}\right) \\
\overleftarrow{D}_{L} \equiv \frac{1}{2}\left(\overleftarrow{\partial}_{\mu}-\frac{1}{2} \omega_{\mu}^{c d} I \sigma_{c d}+\frac{i}{2} \tilde{g} \tau_{i} \tilde{A}_{i \mu}-\frac{i}{2} \tilde{g}^{\prime} \tilde{B}_{\mu}\right) \gamma^{a} V_{a}^{\mu}
\end{gathered}
$$

where $I$ is $2 \times 2$ unit matrix in the isospin space. The forth and the fifth terms in Eq. (94) are defined by equations:

$$
\begin{gathered}
\bar{e}_{R} \not D e_{R} \equiv \bar{e}_{R}\left(\vec{D}_{e R}-\overleftarrow{D}_{e R}\right) e_{R} \\
\overrightarrow{D D}_{e R} \equiv \frac{1}{2} V_{a}^{\mu} \gamma^{a}\left(\vec{\partial}_{\mu}+\frac{1}{2} \omega_{\mu}^{c d} \sigma_{c d}+i \tilde{g}^{\prime} \tilde{B}_{\mu}\right) \\
\overleftarrow{\not D}_{e R} \equiv \frac{1}{2}\left(\overleftarrow{\partial}_{\mu}-\frac{1}{2} \omega_{\mu}^{c d} \sigma_{c d}+-i \tilde{g}^{\prime} \tilde{B}_{\mu}\right) \gamma^{a} V_{a}^{\mu} \\
\bar{\nu}_{R} \not D \nu_{R} \equiv \bar{\nu}_{R}\left(\overrightarrow{\not D}_{\nu R}-\overleftarrow{D D}_{\nu R}\right) \nu_{R} \\
\overrightarrow{D D}_{\nu R} \equiv \frac{1}{2} V_{a}^{\mu} \gamma^{a}\left(\vec{\partial}_{\mu}+\frac{1}{2} \omega_{\mu}^{c d} \sigma_{c d}\right) \\
\overleftarrow{D}_{\nu R} \equiv \frac{1}{2}\left(\overleftarrow{\partial}_{\mu}-\frac{1}{2} \omega_{\mu}^{c d} \sigma_{c d}\right) \gamma^{a} V_{a}^{\mu}
\end{gathered}
$$

In the kinetic term of the scalar fields in Eq.94 the notations are the following:

$$
\begin{gathered}
\left|D_{\mu} \varphi\right|^{2} \equiv\left(D_{\mu} \varphi\right)_{i}^{\dagger}\left(D_{\nu} \varphi\right)^{i} g^{\mu \nu} \\
D_{\mu} \varphi \equiv\left(\partial_{\mu}-\frac{i}{2} \tilde{g} \tau_{i} \tilde{A}_{i \mu}-\frac{i}{2} \tilde{g}^{\prime} \tilde{B}_{\mu}\right) \varphi
\end{gathered}
$$

The gauge complex $u$, which enters into Eq. (94) as an argument of the function $f$ in the second term, is defined now as follows:

$$
u \equiv \frac{1}{4} \tilde{F}_{\mu \nu} \tilde{F}^{\mu \nu}+\frac{1}{2} \operatorname{Tr} \tilde{G}_{\mu \nu} \tilde{G}^{\mu \nu}+\frac{m^{2}}{\sqrt{-g}} \varepsilon^{\mu \nu \alpha \beta} \partial_{\mu} A_{\nu \alpha \beta}
$$

where $\tilde{F}_{\mu \nu} \equiv \partial_{\mu} \tilde{B}_{\nu}-\partial_{\nu} \tilde{B}_{\mu}, \quad \tilde{G}_{\mu \nu} \equiv \partial_{\mu} \tilde{A}_{\nu}-\partial_{\nu} \tilde{A}_{\mu}-i \tilde{g}\left[\tilde{A}_{\mu} \tilde{A}_{\nu}-\tilde{A}_{\nu} \tilde{A}_{\mu}\right]$ where $\tilde{A}_{\mu}=\tilde{A}_{i \mu} T_{i}$.

¿From the action (94) we will come to equations of motion and the constraint similar to what we got before but with the appropriate modifications related to the $S U(2) \times U(1)$ theory. Nonabelian structure of the tensor $\tilde{G}_{\mu \nu}$ does not change the constraint since nonlinear term of $\tilde{G}_{\mu \nu}$ has the same degree of homogeneity in $g^{\mu \nu}$ as the linear terms. After we will get equations similar to Eqs. 39), (85), (51) and (52), we see that $\frac{d V_{\text {eff }}}{d \varphi}=0$ (without a fine tuning of the original potential $V(\varphi)$ ), may be realized when $|\varphi|^{2}=\varphi_{0}^{2}=\frac{1}{2} \eta^{2}$ for some vacuum expectation value

$$
\langle\varphi\rangle=\frac{1}{\sqrt{2}}\left(\begin{array}{l}
0 \\
\eta
\end{array}\right)
$$


Then the appropriate gauge complex condensate $u_{0}$ is determined by Eq. (54) in such a way that the effective cosmological constant defined by Eq. (52) is equal to zero.

Around this vacuum the constraint and equations of motion take the standard form. It is important to note a few remarkable features of the theory. First, in spite of the fact that more than one gauge vector field enter in the gauge complex $u$ (Eq. (106)), the equation similar to Eq. (39) again is enough to provide in the Einstein frame, the form similar to Eq. (47) for all of the gauge field equations. Second, once again, in order to provide the canonical form (47) of the gauge field equations, we have to perform the rescaling

$$
\begin{array}{r}
\vec{A}_{\mu} \equiv 2 \sqrt{\omega} \tilde{\vec{A}}_{\mu} ; \quad B_{\mu} \equiv 2 \sqrt{\omega} \tilde{B}_{\mu} ; \\
g=\frac{\tilde{g}}{2 \sqrt{\omega}} ; \quad g^{\prime}=\frac{\tilde{g}^{\prime}}{2 \sqrt{\omega}}
\end{array}
$$

where the integration constant $\omega$ appears as the universal parameter. It is interesting that with this rescaling, where $\tilde{g} \tilde{A}_{i \mu}=g A_{i \mu}, \tilde{g}^{\prime} \tilde{B}_{\mu}=g^{\prime} B_{\mu}$, we obtain the canonical form of all of the equations similar to Eqs. (46) - (50) with the appropriate modification to the $S U(2) \times U(1)$ theory. From equations similar to Eq. (47) we obtain masses of vector bosons. After the standard field redefinition from $A_{i \mu}, B_{\mu}$ to intermediate vector bosons $W_{\mu}^{+}, W_{\mu}^{-}, Z_{\mu}$ and electromagnetic field $A_{\mu}$, we obtain the following expressions for their masses:

$$
m_{W}=\frac{1}{2} g \eta=\frac{\tilde{g}}{4 \sqrt{\omega}} \eta ; \quad m_{Z}=\frac{1}{2} \bar{g} \eta ; \quad m_{A}=0,
$$

where

$$
\bar{g}=\sqrt{g^{2}+g^{\prime 2}}=\frac{1}{2 \sqrt{\omega}} \sqrt{\tilde{g}^{2}+\tilde{g}^{\prime 2}}
$$

Turning now to the fermionic sector, once again we can find the spin-connection $\omega_{\mu}^{c d}$. Varying the action with respect to $\omega_{\mu}^{c d}$ we obtain an equation similar to Eq. ( $\mathrm{F2}$ ) and the appropriate modification of Eqs. ( $\left.\mathrm{F} 4\right)-(\mathrm{F} 7)$ as the solution of it. In the Einstein frame the $\sigma$-contribution $K_{\mu}^{c d}(\sigma)$ disappears as we discussed at the end of Sec. IVA. Contribution of the fermions selfinteraction to $\omega_{\mu}^{\prime a b}$, like $\propto \kappa \eta_{c i} V_{d \mu}^{\prime} \varepsilon^{a b c d} \overline{e_{L}^{\prime}} \gamma^{5} \gamma^{i} e_{L}^{\prime} \quad\left(e_{L}^{\prime}\right.$ is the left electron spinor in the Einstein frame) which are suppressed by factor $\kappa=16 \pi G$ in comparison to the first term in Eq. (84), may be neglected if we are interested in particle physics at energies much less than the Planck energy scale. Therefore neglecting the last term in Eq. (84) we remain only with the Riemannian contribution to the spin-connection.

Concerning the mass generation of fermions, we have to point out that the choice of the last term in Eq. (94) is related to our intention to use the mechanism for the electron mass generation developed in Sec.IVC, where we have found out that the exponent $q$ must be equal to 2/3. Comparing with notations of Sec.IVC we see that after the SSB, the factor $C$ in Eq. (93) has to be identified in the low energy theory as

$$
k m^{2}=C=\lambda_{e}\left(\frac{\eta}{\sqrt{2}}\right)^{2 / 3} m^{4 / 3}
$$

As a result we get from Eq. (92):

$$
m_{e}=\frac{\lambda_{e}^{3 / 2}}{\sqrt{6 u_{0} \omega}} \eta
$$

Notice that ratios between masses of all particles of the model (see Eq. (55) for the Higgs boson mass, Eqs. (108)), (109) for $W_{\mu}^{ \pm}$and $Z_{\mu}$ masses and the electron mass, Eq. (111)) are $\omega$-independent. The same is true for the weak angle

$$
\sin \theta_{W}=\frac{g^{\prime}}{\bar{g}}
$$

It is very interesting that a big value of the integration constant $\omega$ pushes gauge coupling constants and all masses to small values. In addition, the constant $g_{Y}$ of the effective Yukawa coupling $g_{Y} \bar{L}^{\prime} e_{R}^{\prime} \varphi$

$$
g_{Y}=\frac{\lambda_{e}^{3 / 2}}{\sqrt{3 u_{0} \omega}}
$$


has an additional factor $u_{0}^{-1 / 2}$, which can explain (if the gauge complex condensate $u_{0}=\frac{y_{0}}{m^{4}}$ is also big) the observed further suppression of the effective Yukawa coupling and therefore the appropriate suppression of the observed lepton masses. We can think about all these effects related to a big values of $\omega$ and $u_{0}$ as a "cosmological seesaw mechanism", where masses are driven to small values due to the appearance of large number in denominators.

As we pointed out at the beginning of this section, other gauge unified theories can be formulated in the same fashion. If for example we would consider also QCD, then the same effect of additional suppression would be obtained for the masses of quarks.

\section{THE TRUE VACUUM ENERGY DENSITY AS AN EFFECT OF THE SGMF SYMMETRY BREAKING}

In the previous section we have seen that the presence of the standard cosmological constant term in the original action of the weak NGVE theory does not change the result of the strong NGVE theory: the vacuum energy density of the TVS (in the Einstein picture) is zero. We are going to show now that the appearance of the nonzero vacuum energy density in the TVS (that is the effective cosmological term) in the Einstein picture can be the result of the explicit breaking of the SGMF symmetry (6) or (8) by adding the simplest form of a SGMF symmetry breaking term (9) to the action of the form (7). Since such term is invariant under the LES, it does not contribute to the constraint as one can check explicitly, and therefore the constraint coincides with Eq. (66).

So, let us consider the model with the action

$$
S=\int \Phi d^{4} x\left[-\frac{1}{\kappa} R(\Gamma, g)+\frac{1}{2} \varphi_{, \alpha} \varphi^{, \alpha}-V(\varphi)\right]+\int \Lambda \sqrt{-g} d^{4} x-\gamma \int \frac{\Phi^{2}}{\sqrt{-g}} d^{4} x
$$

Variation with respect to the measure fields $\varphi_{a}$ leads to the equation

$$
A_{a}^{\mu} \partial_{\mu}\left[-\frac{1}{\kappa} R(\Gamma, g)+\frac{1}{2} \varphi_{, \alpha} \varphi^{, \alpha}-V(\varphi)-2 \gamma \frac{\Phi}{\sqrt{-g}}\right]=0
$$

Similar to the cases of the strong as well as the weak NGVE theory, it follows from Eq. (115) that if the measure $\Phi \neq 0$,

$$
-\frac{1}{\kappa} R(\Gamma, g)+\frac{1}{2} \varphi_{, \alpha} \varphi^{, \alpha}-V(\varphi)-2 \gamma \chi=M
$$

Varying the action Eq. (114) with respect to $g^{\mu \nu}$ we get

$$
-\frac{1}{\kappa} R_{\mu \nu}(\Gamma)+\frac{1}{2} \varphi_{, \mu} \varphi_{, \nu}-\left[\frac{\Lambda}{2 \chi}+\frac{\gamma}{2} \chi\right] g_{\mu \nu}=0
$$

¿From Eqs. (116) and (117) we obtain the constraint which coincides in the form with the constraint (66) of Sec. IIIB. The scalar field $\varphi$ equation has the form of Eq. (33).

In the Einstein frame which still is defined by the conformal transformation (44), after using the constraint (66), we get the canonical form of equation for the scalar field (67) with $V_{e f f}^{\prime}=\frac{1}{2 \Lambda}[M+V(\varphi)] V^{\prime}$ and of the gravitational equations in the Riemannian space-time with metric $\bar{g}_{\alpha \beta}$

$$
R_{\mu \nu}(\bar{g})-\frac{1}{2} \bar{g}_{\mu \nu} R(\bar{g})=\frac{\kappa}{2}\left\{\phi,_{\mu} \phi, \nu-\frac{1}{2} \bar{g}_{\mu \nu} \phi, \alpha \phi, \beta \bar{g}^{\alpha \beta}+\left[\frac{1}{4 \Lambda}(V+M)^{2}+\gamma\right] \bar{g}_{\mu \nu}\right\}
$$

In the TVS $\varphi=\varphi_{0}$ where $V\left(\varphi_{0}\right)+M=0$ the last term in Eq.(118) acts as an effective cosmological term.

\section{MODEL WITH CONTINUOUS SYMMETRY RELATED TO THE NGVE PRINCIPLE AND SSB WITHOUT GENERATING A MASSLESS SCALAR FIELD}

It is interesting to see what happens in the above models for the choice $V=J \phi$, where $J$ is some constant. For simplicity we consider here this model in the context of the weak NGVE theory. Then the action (64) is invariant (up to an integral of a total divergence) under the shift $\phi \rightarrow \phi+$ const which is in fact the symmetry $V \rightarrow V+$ const related to the NGVE principle. Notice that if we consider the model with complex scalar field $\psi$, where $\phi$ is the phase of $\psi$, then the symmetry $\phi \rightarrow \phi+$ const would be the $U(1)$ - symmetry of the classical equations of motion. 
The effective potential $V_{e f f}(\phi)=\frac{1}{4 \Lambda}[M+V(\phi)]^{2}$ in such a model has the form

$$
V_{e f f}=\frac{1}{2} m^{2}\left(\phi-\phi_{0}\right)^{2}
$$

where $\phi_{0}=-M / J$ and $m^{2}=J^{2} / 2 \Lambda$. We see that the symmetry $\phi \rightarrow \phi+$ const is spontaneously broken and mass generation is obtained. However, no massless scalar field results from the process of SSB in this case, i.e. Goldstone theorem does not apply here. Of course the conditions under which this theorem was proved do not apply here. In particular, the consideration of a dynamical measure opens up a totally different way to spontaneously break the $U(1)$ symmetry.

This seems to be a special feature of the NGVE - theory which allows: 1) To start with linear potential $J \phi$ without destroying the shift symmetry $\phi \rightarrow \phi+$ const, present in the $\partial_{\mu} \phi \partial^{\mu} \phi$ piece, due to the coupling to the dynamical measure (1). This shift symmetry is now a symmetry of the action up to a total divergence. 2) This potential gives rise to an effective potential $(M+J \phi)^{2} / 4 \Lambda$. The constant of integration $M$ being responsible for the SSB.

In the model of Sec.VI for the choice $V=J \phi$, the only difference is the appearance of the $\lambda$-term in addition to Eq.(119). Similar effect can be obtained also in the strong NGVE - theory but with the use of 4-index field strength condensate. The possibility of constructing spontaneously broken $U(1)$ models which do not lead to associated Goldstone bosons is of course of significant physical relevance. One may recall for example the famous $U(1)$ problem in QCD [21]. Also the possibility of mass generation for axions is of considerable interest. These issues will be developed further in elsewhere.

\section{DISCUSSION AND CONCLUSIONS}

1. Local Einstein Symmetry, Constraint and Degrees of Freedom. In this paper we have seen that the idea to allow the measure to be determined dynamically rather than postulating it to be $\sqrt{-g}$ from the beginning, has deep consequences. In fact, in the context of the first order formalism the NGVE theory does not have a CCP. This is due to the fact that the constraint appears which plays a fundamental role in the theory. With respect to the scalar field $\chi$ which is built from the dynamical measure $\Phi$ and the GR measure $\sqrt{-g} \quad\left(\chi=\frac{\Phi}{\sqrt{-g}}\right)$, the theory behaves in three different ways depending on the structure and symmetries of the action:

i) If we start from the action (5), that is working in the framework of the strong NGVE theory, and the Lagrangian density is such that the constraint is automatically satisfied, then no information about $\chi$ is obtained and as it turns out, in this case there is a so called local Einstein symmetry (29), (30) which allows us to set $\Phi$ to whatever we want and in particular $\Phi=\sqrt{-g}$ is possible.

ii) The second possibility exists, as we have seen in Sec. IIC, where $\chi$ does not enter the constraint, which imposes a condition on other fields of the theory.

iii) In the context both the strong and the weak NGVE theory, we presented several models where the dynamical measure $\Phi$ enters in the constraint (through the field $\chi$ ) and as a result, it is possible to solve the field $\chi$ in terms of other fields. It is very important to note that in such models the constraint describes a connection between the geometrical object (dynamical measure $\Phi$ ) and matter fields, without the Newton constant. This points out to the fact that the constraint affects the physical processes at all region of energy, including low energy physics.

Although we allow the dynamical measure $\Phi$ to carry independent degrees of freedom (from the variational point of view), in all the listed cases after the equations of motions are analyzed, it turns out that the dynamical measure $\Phi$ does not introduce new degrees of freedom. Instead, it is responsible for the rearrangement of interactions in such a way that the CCP problem is solved when the theory is put (by the appropriate change of variables) in the Einstein form where both gravitational constant and all masses are constant. Side by side with the solution of the CCP problem, the possibility to get the exact GR form of equations seems to be a very important feature of the NGVE theory when comparing with usual scalar-tensor theories, where if one chooses the Newton constant to be space-time independent (by the use of a change of variables which involves a conformal transformation), then masses are space-time dependent and vice versa. The above remarks concern the examples discussed in this paper but this does not mean that the NGVE theory cannot lead to a non Einstein behavior (see for example Ref. [15]).

Realistic theories are obtained only for the case (iii) as we have seen in examples (Secs. IID, IIIB, IIIC, V, Appendixes $\mathrm{C}$ and $\mathrm{D}$ ). This has been achieved in several ways: in the context of the strong NGVE theory, the introduction of a 4-index field strength makes it possible to solve $\Phi$ from the constraint in terms of $g_{\mu \nu}$ and matter fields. In the context of the weak NGVE theory, the presence of terms in $L_{2}$ which enter the constraint, makes it possible to solve $\Phi$, again in terms of $g_{\mu \nu}$ and matter fields. 
2. The use of the 4-index field strength in the strong NGVE theory. If a four index field strength is introduced in a 4-D space - time, it develops a condensate which turns out to be expressed in terms of other fields. As a consequence of this, there is the possibility to produce the standard particle physics and gravity dynamics. The resulting dynamics has then interesting consequences in what concerns to the hierarchy problem. As we have seen, all masses and gauge coupling constants are driven to small values if the integration constant $\omega$, that parameterizes the condensate strength (see Eq. (39)), is big. In addition to this, masses of fermions are driven to small values in comparison with masses of bosons as the gauge complex condensate $y_{0}$ becomes big (see Eq. (113)).

The appearance of the parameter $\omega$ in the relation between the original coupling constants and the effective ones suggests an idea that it may be possible to think in different ways concerning renormalization theory. It seems to promise allure prospects since the strength of the condensate specifies a boundary condition or state of the Universe.

It is very interesting that a theory designed in order to solve the cosmological constant problem tells us about an apparently unrelated subject, like what determines the effective coupling constants and masses of the theory. One should recall that the wormhole approach to the cosmological constant problem ends up claiming that wormholes determine all couplings of the theory also [22].

Other important consequence of the theory described in this paper is that one can obtain scalar field dynamics which allows for an inflationary era, the possibility of reheating after scalar field oscillations and the setting down to a zero cosmological constant phase at the later stages of cosmological evolution, without fine tuning. It is interesting that the model not just reproduces all possibilities well known in the cosmology of the very early universe solving at the same time the cosmological constant problem. In addition to this, the effective scalar field potential includes integration constants - a feature which makes us hope that by an appropriate choice of those constants, the correct density perturbations and reheating could be obtained naturally. Moreover, the integration constant $\omega$ enters both in the effective potential and in the effective coupling constants and masses. This means that may be a strong correlation between coupling constants and masses of particles and some of the cosmological parameters.

Some open problems are of course apparent. For example, the "persistent gauge field condensate scenario" (Sec. IID) has to be understood in a deeper way. There a nonspecified function $f$ of a special combination $y$ (Eqs. (34) or (106) as examples) of all gauge fields, including 3-index potential, is introduced. For this function we require only the existence of an extremum at some point $y=y_{0}>0$ and this is enough to get the effective action of electro-weak, QCD and other gauge unified models. The possible origin of such structure as well as the choice of function has to be studied.

The fact that similar type of function appears for example in the QCD effective action as the result of radiative corrections [23], is encouraging. In such a case no four index field strength is introduced however and the effective action is a function of $F_{i \mu \nu} F_{i}^{\mu \nu}$ there. Notice that appearance of a four index field strength condensate in the framework of the theory developed in this paper, makes the Lorentz invariance of the vacuum in QCD not a problem, as opposed to the situation where only regular gauge fields are present as the argument of the nontrivial function, leading to an expectation value of $F_{i \mu \nu} F_{i}^{\mu \nu}$

The four index field strength plays the central role in this model. In this connection, one has to recall that four index field strength plays a fundamental role in some supergravity models, in particular in the $D=11, N=1$ supergravity and in the $D=4, N=8$ supergravity theories. The possibility of incorporating some versions of supergravity into the framework developed in this paper seems therefore a subject which could be a potentially fruitful one.

3. The weak NGVE theory. The very interesting effect we observe in the weak NGVE theory (Sec. III). The addition to the strong NGVE action (5) an explicit cosmological constant term of the canonical GR form $\int \Lambda \sqrt{-g} d^{4} x$ not only does not prevent from the resolution of the CCP in a way similar to what was done in the context of the strong NGVE theory, but it also delivers us from the need to incorporate the four index field strength in order to get a nontrivial resulting dynamics. Moreover, such an explicit cosmological constant term results in the effective potential of the form (71) which for any analytic original potential $V(\varphi)$ and for $\Lambda>0$ allows for the scalar field $\varphi$ to reach in its dynamical evolution the TVS with zero energy density while no fine tuning is needed. Of course, the cosmological consequences of the weak NGVE theory developed in Sec. III are similar to those of the strong NGVE theory with the four - index field strength. However, the absence of the integration constant $\omega$ (Eq. 39) does not allow a strong correlation between coupling constants and masses of particles and some of the cosmological parameters as it is expected in the strong NGVE theory with the four - index field strength.

4. The TVS with nonzero energy density and SGMF symmetry breaking. In order to generate a nonzero energy density in the TVS one has to break the infinite dimensional SGMF symmetry (6) or (8) in the original action. In the simplest model (Sec. VI) this is achieved by adding a SGMF symmetry breaking term (9). If this violation of the SGMF symmetry appears as a result of quantum corrections, it represents then the appearance of an anomaly of the SGMF symmetry (6) or (8). We then have a reason for the smallness of such terms if not of their absolute vanishing ( in the case of exact symmetry). This resembles the situation of quark masses and chiral invariance (CI). In this case, as it is well known, CI forbids a quark mass. If a quark mass nevertheless appears, CI ensures that quark masses remain small even after the consideration of quantum corrections. In a similar way if a small SGMF 
symmetry breaking term appears, hopefully, it will not be renormalized into a large contribution after quantum effects are considered.

5. Remarks concerning the "energy - volume complementarity" and on quantization It is interesting that the mechanism that is responsible for canceling the vacuum energy, which requires $\chi \equiv \Phi / \sqrt{-g} \rightarrow \infty$ as we approach the TVS, can give also some dramatic effects when we consider high energy - densities states. To see this, let us take as an example the model of Sec. IIIB where Eq. (66) gives $\chi \propto(V+M)^{-1}$ (consideration of any of the other examples of Sec. IID, and Appendixes C, D lead to the same qualitative conclusions).

As $V+M \rightarrow 0, \chi \rightarrow \infty$ as mentioned before and this corresponds to the TVS with zero energy density. In the case of high energy density, that is for example allowing formally $V+M \rightarrow \infty$, we obtain that $\chi \equiv \Phi / \sqrt{-g} \rightarrow 0$, in the context of this classical model. Since $\Phi d^{4} x$ has the interpretation of a volume element in the NGVE theory as we have discussed in Introduction and $\sqrt{-g} d^{4} x$ is a standard volume element of GR, we see that where energy densities go to infinity, the volume of the NGVE theory becomes zero relative to the GR volume.

If we were study the behavior of $\chi \equiv \Phi / \sqrt{-g}$ near a high concentration of energy of a point-like particle for example, the same effect is generally obtained, that is the NGVE - volume element goes to zero at short distances from such particle.

This type of effect is generic to a wide class of models mentioned before and in addition to other cases including also models of extended objects ${ }^{3}$ which provide regions with singular energy density where the constraint generally tells us $\chi \rightarrow 0$ as energy density approaches infinity. We will call this effect the "energy - volume complementarity" which means that in a certain sense space - time tries "to run away" from regions of extremely high energy density.

This suggests that in the quantum theory, working with $\Phi, g_{\mu \nu}$ (and not with the effective Einstein variable $\bar{g}_{\alpha \beta}=\chi g_{\alpha \beta}$, undefined for $\chi=0$ ), an interesting effect takes place: where ultra high energy quantum fluctuations occur, space - time volume approaches zero, thus damping in the sense of regularization of those very high excitations.

All this indicates that the correct variables in the high energy regime are the original metric $g_{\alpha \beta}$, the connections $\Gamma_{\mu \nu}^{\lambda}$, the measure fields $\varphi_{a}$ (and the matter fields of course).

In the states which allow regions with $\Phi=0$, which, as we have seen, enter in the complete set of solutions, only $g_{\alpha \beta}$ (and not $\bar{g}_{\alpha \beta}$ ) can be used as the dynamical variable. There and only there the infinite dimensional SGMF symmetry (6) or (8) becomes nontrivial since $L$ or $L_{1}$ are not necessarily constants: if $\Phi=0$, then the equation of the type of Eq. (17) does not imply $\partial_{\mu} L=0$ or $\partial_{\mu} L_{1}=0$ (see Eqs. (13) and (7) for notations). These states therefore play a crucial role for restrictions on the quantum dynamics since much of the structure of the theory is restricted by the existence of the SGMF symmetries (6) or (8).

Summarizing the previous discussion we conclude that states for which $\Phi=0$ is achieved somewhere, correspond to states in the extreme energy density region and the SGMF symmetries (6) or (8) act non trivially. In the quantum theory, we expect therefore that the requirement that such symmetries be preserved by, for example, certain regularizations of the theory, will ensure the preservation of the structure of the theory which is responsible for the solution of the CCP.

Notice finally that in the context of the weak NGVE principle, the infinite dimensional SGMF symmetry still exists in the form specified in Eq. (8). This suggests that the weak NGVE model can be a realization of the effective action that takes into account quantum corrections.

6. A complete group-theoretical and/or algebraic study of the infinite dimensional symmetries of the theory has not been carried out yet and this should be an interesting exercise. One should notice that in addition to (6) or (8) there is the infinite dimensional symmetry of volume preserving internal diffeomorphisms (VPD) $\varphi_{a}^{\prime}=\varphi_{a}^{\prime}\left(\varphi_{b}\right)$ such that $\Phi^{\prime}=\Phi$. Such transformations applied after a transformation of the form (6) or (8) lead to something new. The full symmetry group of transformation contains element that are not in SGMF and not in VPD which are only subgroups of the yet unknown full group of internal symmetries of the measure scalars $\varphi_{a}$.

7. In our treatment of the symmetry $L \rightarrow L+$ const. we have ignored possible topological effects, since $\int \Phi d^{4} x$ being a total divergence can be responsible however for topological effects, similar to the well known $\Theta$-term in QCD.

As we have seen in Sec. VII, such symmetry can be exploited to construct (through the introduction of a linear potential $V=J \phi$ coupled to the measure $\Phi)$ a theory that is globally $U(1)$-invariant with SSB and yet without appearance of a Goldstone boson. Possible applications to axion mass generation, etc has to be explored.

\footnotetext{
${ }^{3}$ Notice that when $\Phi \neq 0$ then $L=$ const. $\quad$ (or $L_{1}=$ const.) and the symmetry (8) (or (8)) represents just a trivial shift of $\varphi_{a}$ by a constant. This shift becomes nontrivial if we allow for points where $\Phi=0$ and therefore $L$ or $L_{1}$ can vary in such "defect" points. Such defects can be naturally associated with extended objects and the nontrivial symmetry (6) or (8) appears then like invariance under reparametrization of the extended object coordinates $\varphi_{a}$.
} 


\section{ACKNOWLEDGMENTS}

We thank Profs. J. Bekenstein, R. Brustein, A. Davidson, A. Dolgov, S. Elitzur, A. Guth, F. Hehl, P. Mannheim, Y. Ne'eman, E. Nissimov, S. Pacheva and R. Zalaletdinov for useful and encouraging discussions on the subjects of this paper.

\section{APPENDIX A: CONNECTION TO OTHER DEVELOPMENTS CONCERNING VOLUME PRESERVING DIFFEOMORPHISMS}

It is interesting to compare the composite density (4) and the form of the equations of motion obtained from the variation of the action (5), with other composite structures and their associated equations of motion.

The simplest instance of a composite structure is obtained by considering two scalar fields $\varphi_{i}(i=1,2)$ and defining the composite field strength [24]

$$
F_{\mu \nu}=\epsilon_{i j} \partial_{\mu} \varphi_{i} \partial_{\nu} \varphi_{j}
$$

where $\epsilon_{i j}$ is the two dimensional antisymmetric symbol with $\epsilon_{12}=1=-\epsilon_{21}, \epsilon_{11}=\epsilon_{22}=0$. The form (A1) derives from a vector potential

$$
F_{\mu \nu}=\partial_{\mu} A_{\nu}-\partial_{\nu} A_{\mu}
$$

without need of imposing an additional condition, for example with

$$
A_{\mu}=\frac{1}{2} \epsilon_{i j} \varphi_{i} \partial_{\mu} \varphi_{j},
$$

all without reference to the dimensionality of space - time.

The form (A1) is associated with an area, that is, a two dimensional volume. In fact, $F_{\mu \nu}$ given by (A1) is invariant under the area preserving diffeomorphisms, i. e. internal transformations in the $\varphi_{i}$ space

$$
\varphi_{i} \rightarrow \varphi_{i}^{\prime}=\varphi_{i}^{\prime}\left(\varphi_{j}\right)
$$

which satisfy the area preserving condition

$$
\epsilon_{i j} \frac{\partial \varphi_{i}^{\prime}}{\partial \varphi_{k}} \frac{\partial \varphi_{j}^{\prime}}{\partial \varphi_{l}}=\epsilon_{k l}
$$

It is not a surprise then that under such transformation $A_{\mu}$ is transformed by the addition of a gradient [24]

$$
A_{\mu} \rightarrow A_{\mu}+\partial_{\mu} \Xi
$$

where $\Xi$ is determined by the area preserving transformation (A4).

One can realize then many of the concepts standard in gauge theories (including minimal coupling to charged matter), where now the gauge symmetry is replaced by the area preserving diffeomorphisms. The number of scalars $\varphi_{i}$ can be enlarged from 2 to $2 n(n=1,2, \ldots)$ and then $F_{\mu \nu}=\omega_{a b} \partial_{\mu} \varphi_{a} \partial_{\nu} \varphi_{b}$ where $\omega_{a b}$ has to be a $2 n \times 2 n$ block diagonal antisymmetric matrix, being equal to $\epsilon_{a b}$ in each block 24. An interesting case in $4-D$ is when we deal with 4 scalars $\varphi_{a}(a=1,2,3,4)$ and

$$
\omega_{a b}=\left(\begin{array}{cccc}
0 & 1 & 0 & 0 \\
-1 & 0 & 0 & 0 \\
0 & 0 & 0 & 1 \\
0 & 0 & -1 & 0
\end{array}\right)
$$

as we will see.

If we take the Lagrangian density in a flat four dimensional space - time as

$$
L=-\frac{1}{4} F_{\mu \nu}\left(\varphi_{a}\right) F^{\mu \nu}\left(\varphi_{a}\right)
$$


and the action $S=\int L d^{4} x$, then we get from the variation with respect to $\varphi_{a}$ the equations

$$
\partial_{\alpha} \varphi_{a}\left(\partial_{\beta} F^{\beta \alpha}\right)=0
$$

For the purpose of comparing with the NGVE theory, let us consider the situation with $n=2$. In this case $\operatorname{det}\left(\partial_{\alpha} \varphi_{a}\right)$ is well defined in $4-D$ space - time. In fact the condition that (A8) is equivalent to Maxwell's equation is that $\Phi \neq 0$ with $\Phi$ being defined by Eq. (4). It has been shown 24 that solutions where $\Phi=0$ exist and they can produce effects like mass generation etc.. As we are pointing out in the present paper, the $\Phi=0$ solutions in the NGVE theory are expected to play an important role and the fact that similar type of solution have been found in a simpler theory, gives us hope that future research in this subject has good possibilities.

Composite gauge fields of the type discussed here have also been found useful applications in the context of brane theory [25]. Further discussion on interrelations between the NGVE theories, composite gauge theories and other developments can be found in [26].

\section{APPENDIX B: METRIC - AFFINE FORMALISM IN THE NGVE THEORY AND $\lambda$-SYMMETRY}

In Sec. IIB we have shown that in the NGVE theory there is a contribution of the $\chi$-field to the connection (see Eqs. (23) and (26)). This contribution is defined up to the $\lambda$-symmetry transformation (27). By using this symmetry, in Sec.IIB we have chosen the gauge where the antisymmetric part of the connection (that is a $\chi$-contribution into the torsion) disappears.

It is interesting to see what is the geometrical meaning of the $\lambda$-gauge dependent contribution to the connection (23), (26). For this we calculate the covariant derivative of the metric tensor $g_{\mu \nu}$ with the connection defined by (23) and (26) and we get

$$
g_{\mu \nu ; \alpha}=-2 g_{\mu \nu} \lambda_{, \alpha} \equiv N_{\mu \nu \alpha} .
$$

This means that the $\lambda$ dependent contribution to the connection is responsible for the appearance of the nonmetricity tensor 27.

With the choice $\lambda=\frac{1}{2} \sigma$, in Sec. IIB, we have eliminated the $\chi$-contribution into the torsion keeping the nonmetricity tensor $N_{\mu \nu \alpha}$ which in this " $\sigma$-torsionless" gauge is equal to $-g_{\mu \nu} \sigma_{, \alpha}$. However we have the freedom to choose for example the " $\sigma$ - metric - compatible" gauge where the nonmetricity disappears: $\lambda=$ constant. In such a case, the torsion is not eliminated from the connection (23), (26).

Notice that these peculiarities of the $\lambda$-symmetry concerning the possibility of eliminating the $\chi$-contribution to the torsion or, alternatively, of eliminating the $\chi$-contribution to the nonmetricity appear to be a very interesting feature of the NGVE theory in the metric - affine formalism. This feature results from the basic assumption that not only metric and connection are independent dynamical variables (as it is in the case of the Metric - Affine theory), but also the measure degrees of freedom are independent variables when varying the action.

\section{APPENDIX C: FOUR INDEX FIELD STRENGTH CONDENSATE AS AN UNIVERSAL GOVERNOR. SIMPLE MODELS.}

\section{Scalar field + four index field strength}

As it follows from our analysis in Sec.IIC, a model with only a scalar field although solves the cosmological constant problem, it cannot give a nontrivial dynamics. We study here the simplest model which in addition to a single scalar field with a nontrivial potential includes also an additional degree of freedom described by a three-index potential $A_{\beta \mu \nu}$ as in the Lagrangian density

$$
L=-\frac{1}{\kappa} R(\Gamma, g)+\frac{1}{2} \varphi,_{\alpha} \varphi^{, \alpha}-V(\varphi)+\frac{1}{4 !} F_{\alpha \beta \mu \nu} F^{\alpha \beta \mu \nu} .
$$

Here 


$$
F_{\alpha \beta \mu \nu} \equiv \partial_{[\alpha} A_{\beta \mu \nu]}
$$

is the field strength which is invariant under the gauge transformation

$$
A_{\beta \mu \nu} \rightarrow A_{\beta \mu \nu}+\partial_{[\beta} f_{\mu \nu]}
$$

In ordinary 4-dimensional GR, the $F_{\alpha \beta \mu \nu} F^{\alpha \beta \mu \nu}$ term gives rise to a cosmological constant depending on an integration constant [28], [29]. In our case, due to the constraint (20), the degrees of freedom contained in $F_{\alpha \beta \mu \nu}$ and those of the scalar field $\varphi$ will be intimately correlated. The sign in front of the $F_{\alpha \beta \mu \nu} F^{\alpha \beta \mu \nu}$ term is chosen so that in this model the resulting expression for the energy density of the scalar field $\varphi$ is a positive definite one for any possible space-time dependence of $\varphi$ in the Einstein picture". Notice also that two last terms in the action with the Lagrangian (C1) break explicitly the LES.

The gravitational equations (19) take now the form

$$
-\frac{1}{\kappa} R_{\mu \nu}(\Gamma)+\frac{1}{2} \varphi,{ }_{\mu} \varphi,_{\nu}+\frac{1}{6} F_{\mu \alpha \beta \gamma} F_{\nu}^{\alpha \beta \gamma}=0 .
$$

Notice that the scalar field potential $V(\varphi)$ does not appear explicitly in Eqs. (C4). However, the constraint (20), which takes now the form

$$
V(\varphi)+M=-\frac{1}{8} F_{\alpha \beta \mu \nu} F^{\alpha \beta \mu \nu},
$$

allows us to express the last term in ( $\mathrm{C} 4)$ in terms of the potential $V(\varphi)$ (using the fact that $F^{\alpha \beta \mu \nu} \propto \varepsilon^{\alpha \beta \mu \nu}$ in 4-dimensional space-time).

Varying the action with respect to $A_{\nu \alpha \beta}$, we get the equation

$$
\partial_{\mu}\left(\Phi F^{\mu \nu \alpha \beta}\right)=0
$$

Its general solution has a form

$$
F^{\alpha \beta \mu \nu}=\frac{\lambda}{\Phi} \varepsilon^{\alpha \beta \mu \nu} \equiv \frac{\lambda}{\chi \sqrt{-g}} \varepsilon^{\alpha \beta \mu \nu},
$$

where $\lambda$ is an integration constant. Then $F_{\alpha \beta \mu \nu} F^{\alpha \beta \mu \nu}=-\lambda^{2} 4 ! / \chi^{2}$ is not a constant now as opposed to the GR case [28], [29] and therefore

$$
V(\varphi)+M=3 \lambda^{2} / \chi^{2}
$$

and

$$
F_{\mu \alpha \beta \gamma} F_{\nu}^{\alpha \beta \gamma}=-\left(6 \lambda^{2} / \chi^{2}\right) g_{\mu \nu}=-2[V(\varphi)+M] g_{\mu \nu}
$$

This shows how the potential $V(\varphi)$ appears in Eq. (C4), spontaneously violating the symmetry of the action $V(\varphi) \rightarrow$ $V(\varphi)+$ constant, which now corresponds to a redefinition of the integration constant $M$.

The equation of motion of the scalar field $\varphi$ is

$$
(-g)^{-1 / 2} \partial_{\mu}\left(\sqrt{-g} g^{\mu \nu} \partial_{\nu} \varphi\right)+\sigma_{, \mu} \varphi^{\mu}+V^{\prime}(\varphi)=0,
$$

where $V^{\prime} \equiv \frac{d V}{d \varphi}$.

The derivatives of the field $\sigma$ enter both in the gravitational Eqs. (C4) (through the connection) and in the scalar field equation (C10). In order to see easily the physical content of this model, we have to perform a conformal transformation

$$
\bar{g}_{\mu \nu}(x)=\chi g_{\mu \nu}(x) ; \quad \varphi \rightarrow \varphi
$$

to obtain an Einstein picture. Notice that now this transformation is not a symmetry and indeed changes the form of equations. In this new frame, the gravitational equations become

$$
G_{\mu \nu}\left(\bar{g}_{\alpha \beta}\right)=\frac{\kappa}{2} T_{\mu \nu}^{e f f}(\varphi)
$$


where

$$
G_{\mu \nu}\left(\bar{g}_{\alpha \beta}\right)=R_{\mu \nu}\left(\bar{g}_{\alpha \beta}\right)-\frac{1}{2} \bar{g}_{\mu \nu} R\left(\bar{g}_{\alpha \beta}\right)
$$

is the Einstein tensor in the Riemannian space-time with metric $\bar{g}_{\mu \nu}$, and the source is the minimally coupled scalar field $\varphi$

$$
T_{\mu \nu}^{e f f}(\varphi)=\varphi,_{\mu} \varphi,_{\nu}-\frac{1}{2} \bar{g}_{\mu \nu} \varphi,,_{\alpha} \varphi,^{\alpha}+\bar{g}_{\mu \nu} V_{e f f}(\varphi)
$$

with the new effective potential

$$
V_{e f f}=\frac{2}{3} \chi^{-1}(V+M)
$$

The scalar field equation (C10) in the Einstein picture takes a form

$$
\frac{1}{\sqrt{-\bar{g}}} \partial_{\mu}\left(\sqrt{-\bar{g}} \bar{g}^{\mu \nu} \partial_{\nu} \varphi\right)+\chi^{-1} V^{\prime}(\varphi)=0 .
$$

For the possible expression for $\chi^{-1}$ we have from Eq. (C8)

$$
\frac{1}{\chi}= \pm \frac{1}{\lambda \sqrt{3}}(V+M)^{1 / 2}
$$

Independently of the sign chosen for $\chi$ in Eq. (C17), the gravitational and scalar field equations have the same physical content expressed in different space-time signatures. In what follows we simply take the + sign in (C17). Therefore, the effective scalar field potential (C15) has the form

$$
V_{e f f}(\varphi)=\frac{2}{\lambda 3 \sqrt{3}}(V+M)^{3 / 2}
$$

and the scalar field Eq. ( $\overline{\mathrm{C} 16}$ ) becomes a conventional general relativistic scalar field equation with the potential $V_{e f f}(\varphi)$ :

$$
\frac{1}{\sqrt{-\bar{g}}} \partial_{\mu}\left(\sqrt{-\bar{g}} \bar{g}^{\mu \nu} \partial_{\nu} \varphi\right)+V_{e f f}^{\prime}(\varphi)=0
$$

We see that in the Einstein picture, for any analytic $V(\varphi), V_{\text {eff }}(\varphi)$ has an extremum, that is $V_{e f f}^{\prime}=0$, either when $V^{\prime}=0$ or $V+M=0$. The extremum when $V+M=0$ corresponds to an absolute minimum (since $V_{\text {eff }}(\varphi)$ is non negative) and therefore it is a vacuum with zero effective cosmological constant. It should be emphasized that all what is required is that $V+M$ reaches zero at some point $\varphi_{0}$ but $V^{\prime}$ at this point does not need to vanish. Therefore no fine tuning in the usual sense, of adjusting a minimum of a potential to coincide with the point where this potential itself vanishes, is required. And the situation is even more favorable since even if $V+M$ happens not to touch zero for any value of $\varphi$, we always have an infinite set of other values of the integration constant $M$ where this will happen. However, this model is not as satisfactory as those discussed in the text since the second derivative of $V_{\text {eff }}$ is singular in its absolute minimum.

\section{Gauge fields and the Higgs mechanism in the NGVE theory}

Let us consider now a model including gravity, four index field strength $F_{\alpha \beta \mu \nu}$, a gauge field $\tilde{A}_{\mu}$ and a complex scalar field $\phi$ minimally coupled to the gauge field with the action

$$
\begin{array}{r}
S=\int \Phi d^{4} x\left[-\frac{1}{\kappa} R(\Gamma, g)+\frac{1}{4 !} F_{\alpha \beta \mu \nu} F^{\alpha \beta \mu \nu}+\frac{1}{m^{4}}\left(\tilde{F}_{\mu \nu} \tilde{F}^{\mu \nu}\right)^{2}\right. \\
\left.+g^{\mu \nu}\left(\partial_{\mu}-i \tilde{e} \tilde{A}_{\mu}\right) \phi\left(\partial_{\nu}+i \tilde{e} \tilde{A}_{\nu}\right) \phi^{*}-V(|\phi|)\right]
\end{array}
$$


where $\tilde{F}_{\mu \nu} \equiv \partial_{\mu} \tilde{A}_{\nu}-\partial_{\nu} \tilde{A}_{\mu}$.

Notice that the kinetic term of the gauge field $\tilde{A}_{\mu}$ is chosen in an unusual way where an additional parameter $m$ with dimensionality of mass is introduced to provide the canonical dimensionality for the gauge field $\tilde{A}_{\mu}$. The reason for such a choice of the kinetic term of the gauge field is to achieve for it the same degree of homogeneity in $g^{\mu \nu}$ in the Lagrangian density as we have for the four index field strength $F_{\alpha \beta \mu \nu}$. As we will see, for such a choice, after solving the constraint we obtain the standard effective low energy physics. For example, in the absence of other interactions, the gauge field equations possess conformal invariance or, what is the same, they have the standard Maxwell form.

By making use the gauge invariance we choose the unitary gauge (where $\operatorname{Im} \phi(x)=0$ ) and then the Lagrangian density takes the form

$$
\begin{array}{r}
L=-\frac{1}{\kappa} R(\Gamma, g)+\frac{1}{4 !} F_{\alpha \beta \mu \nu} F^{\alpha \beta \mu \nu}+\frac{1}{m^{4}}\left(\tilde{F}_{\mu \nu} \tilde{F}^{\mu \nu}\right)^{2}+ \\
\left.\frac{1}{2} g^{\mu \nu} \varphi,{ }_{\mu} \varphi,{ }_{\nu}-V(\varphi)+\frac{1}{2} \tilde{e}^{2} \varphi^{2} g^{\mu \nu} \tilde{A}_{\mu} \tilde{A}_{\nu}\right)
\end{array}
$$

where we have defined $|\phi|=\frac{1}{\sqrt{2}} \varphi$.

The constraint (20) corresponding to the Lagrangian density (C21) is

$$
\frac{1}{8} F_{\alpha \beta \mu \nu} F^{\alpha \beta \mu \nu}+\frac{3}{m^{2}}\left(\tilde{F}_{\mu \nu} \tilde{F}^{\mu \nu}\right)^{2}+V(\varphi)+M=0
$$

Similar to the first and the fourth terms, the last term in Eq. (C21) does not contribute to the constraint since it is homogeneous of degree one in $g^{\mu \nu}$.

The equation for $F_{\alpha \beta \mu \nu}$ and its solution are still the same as in Eqs. (C6), (C7) which bring constraint (C22) to the following equation:

$$
\frac{\omega^{2} m^{4}}{\chi^{2}}=\frac{1}{m^{4}}\left(\tilde{F}_{\mu \nu} \tilde{F}^{\mu \nu}\right)^{2}+\frac{1}{3}(V(\varphi)+M)
$$

where we have defined $\lambda$ in terms of the mass parameter $m^{2}$

$$
\lambda=\omega m^{2},
$$

$\omega$ being a dimensionless constant.

Varying the action with respect to $\tilde{A}_{\mu}$ we get

$$
\frac{1}{\sqrt{-g}} \partial_{\mu}\left[\sqrt{-g} \chi\left(\tilde{F}_{\alpha \beta} \tilde{F}^{\alpha \beta}\right) g^{\mu \gamma} g^{\nu \delta} \tilde{F}_{\gamma \delta}\right]-\frac{\tilde{e}^{2} m^{4}}{8} \varphi^{2} \chi g^{\alpha \nu} \tilde{A}_{\alpha}=0
$$

Looking at gauge field fluctuations around the true vacuum $\varphi=\varphi_{0}$ where $V\left(\varphi_{0}\right)+M=0$ and $V_{\text {eff }}^{\prime}\left(\varphi_{0}\right)=0$ (and ignoring the scalar field fluctuations around the true vacuum $\varphi_{0}$, see the previous subsection), we get from (C23)

$$
\chi\left(\tilde{F}_{\mu \nu} \tilde{F}^{\mu \nu}\right)= \pm \omega m^{4}
$$

First of all notice that in the case where is no coupling to the scalar field ( $\tilde{e}=0)$, Eq. C25) after making use Eq. (C26) becomes the Maxwell's equations

$$
\frac{1}{\sqrt{-g}} \partial_{\mu}\left(\sqrt{-g} g^{\mu \gamma} g^{\nu \delta} \tilde{F}_{\gamma \delta}\right)=0
$$

which are indeed conformally invariant.

In the presence of interactions with scalar field $(\tilde{e} \neq 0)$, Eqs. (C25) and (C26) lead to the singularity in the second term when $\tilde{F}_{\alpha \beta} \tilde{F}^{\alpha \beta}=0$.

Notice that the $\chi$-field becomes divergent as $\varphi$ approaches the absolute minimum $\varphi_{0}$. Therefore it is not a surprise that a singularity also occurs in the case where $\tilde{F}_{\alpha \beta} \tilde{F}^{\alpha \beta}=0$, that is when electric dominated field evolves into magnetic dominated one (or vice versa). Here we will see that this singularity is eliminated by the conformal transformation (C11) to the Einstein frame.

In fact, performing the conformal transformation (C11) and taking into account the constraint (C26) we obtain for the gauge field equation in the Einstein frame 


$$
\frac{1}{\sqrt{-\bar{g}}} \partial_{\mu}\left[ \pm \sqrt{-\bar{g}} \bar{g}^{\mu \gamma} \bar{g}^{\nu \delta} \tilde{F}_{\gamma \delta}\right]-\frac{\tilde{e}^{2}}{8 \omega} \varphi^{2} \bar{g}^{\alpha \nu} \tilde{A}_{\alpha}=0
$$

Notice that we have not taken the sign \pm in Eq. $(\mathrm{C} 28)$ outside the derivative operator. This makes it apparent that if we pick one of the two branches displayed in Eq. (C28), it cannot evolve continuously to the another branch. Tf Therefore the requirement of the analyticity of the resulting equations exclude for example the alternative $\chi\left|\tilde{F}_{\mu \nu} \tilde{F}^{\mu \nu}\right|=$ $\omega m^{4}$ instead of $(\overline{\mathrm{C} 26})$ as a solution of the constraint (C23) at the point $\varphi=\varphi_{0}$.

When choosing one of the branches in Eq. (C26) we note that the conformal transformation (C11) changes the relative signatures of the original metric $g_{\mu \nu}$ and the metric $\bar{g}_{\mu \nu}$ in the Einstein frame when the gauge field evolves from electric dominated to magnetic dominated (and vice versa).

Eq. ( $\mathrm{C} 28$ ) shows that in the Einstein frame there is no singularity when $\chi^{-1} \rightarrow \pm 0$. Therefore, changes of the signature of the metric take place only in the original frame and not in the Einstein frame.

For the choice of the signature $(+---)$ in the Einstein frame we have to choose the branch $(-)$ in Eq. (C28) in order to avoid tachyonic behavior. After the change of notations

$$
e=\frac{\tilde{e}}{2 \sqrt{2 \omega}} ; \quad A_{\mu}=2 \sqrt{2 \omega} \tilde{A}_{\mu} ;
$$

we get the canonical form of equations for the vector field

$$
\frac{1}{\sqrt{-\bar{g}}} \partial_{\mu}\left(\sqrt{-\bar{g}} \bar{g}^{\mu \gamma} \bar{g}^{\nu \delta} F_{\gamma \delta}\right)+m_{A}^{2} A^{\nu}=0
$$

where

$$
m_{A}^{2}=e^{2} \varphi_{0}^{2}
$$

is the mass of the vector boson $A_{\mu}$ which is generated by the spontaneous symmetry breaking (SSB) of the gauge invariance when the scalar field $\phi$ is located at the absolute minimum $|\phi|=\frac{1}{\sqrt{2}} \varphi=\frac{1}{\sqrt{2}} \varphi_{0}$ of the effevtive potential (C18). We have used the notations

$$
A^{\mu}=\bar{g}^{\mu \nu} A_{\nu} ; F_{\mu \nu}=\partial_{\mu} A_{\nu}-\partial_{\nu} A_{\mu} ; F^{\mu \nu}=\bar{g}^{\mu \alpha} \bar{g}^{\nu \beta} F_{\alpha \beta}
$$

The appropriate gravitational equations at the absolute minimum $\varphi_{0}$ in the Einstein frame, when the source is the vector field $A_{\mu}$ takes the standard form

$$
G_{\mu \nu}\left(\bar{g}_{\alpha \beta}\right)=\frac{\kappa}{2} T_{\mu \nu}\left(A_{\alpha}\right)
$$

where

$$
T_{\mu \nu}\left(A_{\alpha}\right)=\frac{1}{4} \bar{g}_{\mu \nu} F_{\alpha \beta} F^{\alpha \beta}-F_{\mu \alpha} F_{\nu \beta} \bar{g}^{\alpha \beta}+\frac{1}{2} m_{A}^{2}\left(A_{\mu} A_{\nu}-\frac{1}{2} \bar{g}_{\mu \nu} A_{\alpha} A^{\alpha}\right)
$$

For the scalar field equation in the Einstein frame we obtain

$$
\frac{1}{\sqrt{-\bar{g}}} \partial_{\mu}\left(\sqrt{-\bar{g}} \bar{g}^{\mu \nu} \partial_{\nu} \varphi\right)-e^{2} A_{\alpha} A^{\alpha} \varphi+V_{e f f}^{\prime}=0
$$

with $V_{\text {eff }}$ given by Eq. (C18).

As we see, the rescaling (C29) provides the canonical normalization of the gauge field so to reproduce the standard form of the energy-momentum tensor (C34) and standard interaction of the vector field $A_{\mu}$ to the scalar field after SSB. A very interesting feature of the theory in the Einstein picture is the fact that the gauge coupling constant $e$ depends on the integration constant $\omega$ which appears in the solution for the four index field condensate (C7) (see also the definition (C24).

Finally we have to notice that it is possible to improve the model discussed in this Sec.IV (see for example [5]) in such a way that the mass of the scalar field becomes finite (compare with discussion at the end of Subsec.IVA). Then, however, at the very high energies where the scalar field fluctuations around the vacuum $\varphi=\varphi_{0}$ have to be taken into account, it follows from the constraint (C23) and Eq. (C25) that the nonminimal nonrenormalizable interaction of the gauge field with the scalar field appears.

\footnotetext{
${ }^{4}$ We have to point out that the models discussed in the body of the paper do not suffer this ambiguity.
} 


\section{APPENDIX D: MODEL WITH A CRITICAL LIMIT}

The aim of this Appendix is to demonstrate reasons which lead us to the model with persistent gauge condensate (Sec.IID). In the framework of the strong NGVE principle we consider here the interesting family of Lagrangians which depend on the ordinary gauge field $\tilde{A}_{\mu}$ and the three index gauge field $A_{\mu \nu \alpha}$ only through the gauge complex $y$ Eq. (34) with the power low dependence on $y$. Therefore, in the unitary gauge for $\tilde{A}_{\mu}$, instead of dealing with (C20), (C21), we have the action

$$
S=\int \Phi d^{4} x\left[-\frac{1}{\kappa} R(\Gamma, g)-\frac{1}{p m^{4(p-1)}} y^{p}+\frac{1}{2} g^{\mu \nu} \varphi, \mu \varphi,_{\nu}-V(\varphi)+\frac{1}{2} \tilde{e}^{2} \varphi^{2} g^{\mu \nu} \tilde{A}_{\mu} \tilde{A}_{\nu}\right]
$$

where dimensionless parameter $p$ is a real number. As we will see later, the physically interesting case is achieved in the critical limit $p \rightarrow \infty$.

Constraint (20) takes now the form

$$
\frac{2 p-1}{p m^{4(p-1)}} y^{p}=V(\varphi)+M
$$

which defines $y$ as a function of $\varphi$.

¿From variation with respect to $A_{\nu \alpha \beta}$ we obtain the equation

$$
\partial_{\mu}\left(\chi y^{p-1} \varepsilon^{\mu \nu \alpha \beta}\right)=0
$$

the solution of which can be written as

$$
\chi y^{p-1}=\omega m^{4(p-1)}
$$

where $\omega$ is a dimensionless integration constant.

Varying with respect to the scalar field $\varphi$ we get

$$
(-g)^{-1 / 2} \partial_{\mu}\left(\sqrt{-g} g^{\mu \nu} \partial_{\nu} \varphi\right)+\sigma, \mu \varphi^{, \mu}+V^{\prime}(\varphi)+\tilde{e}^{2} \varphi g^{\alpha \beta} \tilde{A}_{\alpha} \tilde{A}_{\beta}=0
$$

The equation for the gauge field $\tilde{A}_{\alpha}$ is

$$
\frac{1}{\sqrt{-g}} \partial_{\mu}\left[\chi y^{p-1} \sqrt{-g} \tilde{F}^{\mu \nu}\right]+\frac{\tilde{e}^{2}}{4} m^{4(p-1)} \varphi^{2} \chi \tilde{A}^{\nu}=0
$$

which becomes

$$
\frac{1}{\sqrt{-g}} \partial_{\mu}\left[\sqrt{-g} \tilde{F}^{\mu \nu}\right]+\frac{\tilde{e}^{2}}{4 \omega} \varphi^{2} \chi \tilde{A}^{\nu}=0
$$

due to Eq. (D4). And finally, the variation of $g^{\mu \nu}$ leads to the gravitational equations

$$
\frac{1}{\kappa} R_{\mu \nu}(\Gamma, g)=-\frac{y^{p}}{2 m^{4(p-1)}} g_{\mu \nu}+\frac{y^{p-1}}{m^{4(p-1)}}\left[\frac{1}{2} \tilde{F}^{\alpha \beta} \tilde{F}_{\alpha \beta} g_{\mu \nu}-2 \tilde{F}_{\mu \alpha} \tilde{F}_{\nu \beta} g^{\alpha \beta}\right]+\frac{1}{2} \varphi_{, \mu} \varphi, \nu+\frac{\tilde{e}^{2}}{2} \varphi^{2} \tilde{A}_{\mu} \tilde{A}_{\nu}
$$

where Eq. (34) has been used.

For the same reasons as those explained in Sec. IV, we have to perform the conformal transformation (C11) which provides a formulation of the theory in the Einstein picture. Eqs. (D5), (D7) and (D\&) become then correspondingly

$$
\begin{gathered}
\frac{1}{\sqrt{-\bar{g}}} \partial_{\mu}\left(\sqrt{-\bar{g}} \bar{g}^{\mu \nu} \partial_{\nu} \varphi\right)+\frac{d V_{e f f}^{(p)}}{d \varphi}+e^{2} \varphi g^{\alpha \beta} A_{\alpha} A_{\beta}=0 \\
\frac{1}{\sqrt{-\bar{g}}} \partial_{\mu}\left(\sqrt{-\bar{g}} \bar{g}^{\mu \alpha} \bar{g}^{\nu \beta} F_{\alpha \beta}\right)+e^{2} \varphi^{2} \bar{g}^{\nu \alpha} A_{\alpha}=0 \\
G_{\mu \nu}\left(\bar{g}_{\alpha \beta}\right)=\frac{\kappa}{2} T_{\mu \nu}
\end{gathered}
$$




$$
T_{\mu \nu}=\varphi_{, \mu} \varphi_{, \nu}-\frac{1}{2} g_{\mu \nu} \varphi_{, \alpha} \varphi_{, \beta} \bar{g}^{\alpha \beta}+V_{e f f}^{(p)}(\varphi) \bar{g}_{\mu \nu}+\frac{1}{4} \bar{g}_{\mu \nu} F_{\alpha \beta} F^{\alpha \beta}-F_{\mu \alpha} F_{\nu \beta} \bar{g}^{\alpha \beta}+e^{2} \varphi^{2}\left(A_{\mu} A_{\nu}-\frac{1}{2} \bar{g}_{\mu \nu} A_{\alpha} A^{\alpha}\right)
$$

where

$$
V_{e f f}^{(p)}(\varphi) \equiv\left[\omega m^{4(1-1 / p)}\right]^{-1}\left(\frac{p}{2 p-1}\right)^{2-1 / p}(V(\varphi)+M)^{2-1 / p}
$$

and the rescalings $(45)$ have been performed. It is assumed that $\omega>0$.

Equations (D9)-(D12) describe the family of canonical equations of GR (parameterized by the parameter $p$ ) for a gauge model (in the unitary gauge) including gauge field $A_{\mu}$ minimally coupled to the scalar field $\phi$ with the potential (D13) and the coupling constant $e$. For $p=1$ (which has to be studied by itself), the theory reproduces the Einstein GR with the original potential $V(\varphi)$ and with a cosmological constant $M$ (see Ref. [3]). In contrast, for any $p>1$, the effective potential $V_{e f f}^{(p)}(\varphi)$, given by Eq.(D13), has an absolute minimum at the point $\varphi_{0}$ where $V\left(\varphi_{0}\right)+M=0$.

However an additional remarkable feature appears when $p \rightarrow \infty$. In this case

$$
V_{e f f}(\varphi) \equiv \lim _{p \rightarrow \infty} V_{e f f}^{(p)}(\varphi)=\frac{1}{4 \omega m^{4}}(V+M)^{2}
$$

and for any analytical function $V(\varphi)$, all derivatives of the effective potential $V_{\text {eff }}(\varphi)$ are finite at the absolute minimum $\varphi=\varphi_{0}$ where $V\left(\varphi_{0}\right)+M=0$. In particular, $V_{e f f}^{\prime \prime}\left(\varphi_{0}\right) \propto\left[V^{\prime}\left(\varphi_{0}\right)\right]^{2}$ is finite (and nonzero if we do not carry out the fine tuning $V^{\prime}\left(\varphi_{0}\right)=0$ ). Therefore the Higgs boson, in particular, can reappear as a physical particle of the theory. In the context of cosmology where $V_{\text {eff }}(\varphi)$ plays the role of the inflaton potential, a finite mass of the inflaton allows to recover the usual oscillatory regime of the reheating period after inflation that are usually considered.

Notice that again (as it was in Sec.IIID) the gauge coupling constant $e$ in the Einstein frame (see Eq. (45)) depends on the integration constant $\omega$ which appears in the solution for the four index field condensate (D4).

¿From Eqs.(D2) and (D4) it follows that

$$
\chi=\left(2-\frac{1}{p}\right)^{1-1 / p} \omega m^{4(1-1 / p)}(V+M)^{-1+1 / p}
$$

so that if $p>1$ or $p<0$ we obtain that $\chi \rightarrow \infty$ as $V+M \rightarrow 0$.

It is very instructive to look at what happens to the condensate $y$ when we approach the true vacuum $\varphi=\varphi_{0}$ where $V\left(\varphi_{0}\right)+M=0$. For any finite $p>1$ we see from Eq. (D2) that $y=(V+M)^{1 / p} m^{4-4 / p}\left(\frac{p}{2 p-1}\right)^{1 / p}$ and $y \rightarrow 0$ in this limit. We notice however the very interesting effect which consists of the fact that as $p$ becomes big, $y$ approaches zero but at a very slow rate. In the limit $p \rightarrow \infty$ we can indeed argue that $y$ does not necessarily approaches zero but rather to an undetermined constant since $y \sim 0^{0}$ which is not defined. This suggests that the possible existence of a condensate $y$ that survives even in the true vacuum (which we will call "persistent condensate") is the cause of the remarkable feature which allows $V_{\text {eff }}$ to be of the form (D14). In Sec. IID we have verified this explicitly.

\section{APPENDIX E: CONSTRAINT AND LES IN THE VIERBEIN - SPIN-CONNECTION FORMALISM}

To incorporate fermions into the NGVE theory we have to use the vierbein - spin-connection (VSC) formalism (see, however a purely affine approach due to Ne'eman [30]). In this Appendix we review in a short form how the NGVE principle works in the VSC-formalism.

In this case we define 17]

$$
\begin{gathered}
R(\omega, V)=V^{a \mu} V^{b \nu} R_{\mu \nu a b}(\omega) \\
R_{\mu \nu a b}(\omega)=\partial_{\mu} \omega_{\nu a b}-\partial_{\nu} \omega_{\mu a b}+\left(\omega_{\mu a}^{c} \omega_{\nu c b}-\omega_{\nu a}^{c} \omega_{\mu c b}\right)
\end{gathered}
$$

where $V^{a \mu}=\eta^{a b} V_{b}^{\mu}, \eta^{a b}$ is the diagonal $4 \times 4$ matrix with elements $(1,-1,-1,-1)$ on the diagonal, $V_{a}^{\mu}$ are the vierbeins and $\omega_{\mu}^{a b}=-\omega_{\mu}^{b a}(a, b=0,1,2,3)$ is the spin connection. The matter Lagrangian $L_{m}$ that appears in Eq. (5) is now a function of matter fields, vierbeins and spin connection, considered as independent fields. We assume for simplicity that $L_{m}$ does not depend on the derivatives of vierbeins and spin connection. 
We are now going to study the theory defined by the action (5) in the case that the scalar curvature is defined by (E1), (E2).

As in Sec. IIA, variation with respect to the scalar fields $\varphi_{a}$ leads to the equations

$$
A_{a}^{\mu} \partial_{\mu}\left(-\frac{1}{\kappa} R(V, \omega)+L_{m}(V, \omega, \text { matterfields })\right)=0
$$

which implies, if $\Phi \neq 0$, that

$$
-\frac{1}{\kappa} R(V, \omega)+L_{m}(V, \omega, \text { matterfields })=M
$$

On the other hand, considering the equations obtained from the variation of the vierbeins, we get if $\Phi \neq 0$

$$
-\frac{2}{\kappa} R_{\mu a}(V, \omega)+\frac{\partial L_{m}}{\partial V^{a \mu}}=0
$$

where

$$
R_{\mu a}(V, \omega) \equiv V^{b \nu} R_{\mu \nu a b}(\omega) .
$$

Notice that eq. $(\mathrm{E} 5)$ is indeed invariant under the shift $L_{m} \rightarrow L_{m}+$ const. By using Eq. (E1) we can eliminate $R(\omega)$ from the equations (E4) and (E5) after contracting the last one with $V_{a \mu}$. As a result we obtain the nontrivial constraint in the form

$$
V^{a \mu} \frac{\partial\left(L_{m}-M\right)}{\partial V^{a \mu}}-2\left(L_{m}-M\right)=0
$$

which replaces Eq. 20) (see Sec. II) and in the absence of fermions, is equivalent to the constraint (20).

The simplest example of a fermion is that of spin $1 / 2$ particles. In this case we regard the spinor field $\Psi$ as a general coordinate scalar and transforming nontrivially with respect to local Lorentz transformation according to the spin $1 / 2$ representation of the Lorentz group.

The NGVE principle prescripts for the fermionic hermitian action (which allows for the possibility of fermion self interactions) to be of the form

$$
S_{f}=\int L_{f} \Phi d^{4} x
$$

where

$$
L_{f}=\frac{i}{2} \bar{\Psi}\left[\gamma^{a} V_{a}^{\mu}\left(\vec{\partial}_{\mu}+\frac{1}{2} \omega_{\mu}^{c d} \sigma_{c d}\right)-\left(\overleftarrow{\partial}_{\mu}-\frac{1}{2} \omega_{\mu}^{c d} \sigma_{c d}\right) \gamma^{a} V_{a}^{\mu}\right] \Psi+U(\bar{\Psi} \Psi)
$$

Here $\sigma_{c d} \equiv \frac{1}{4}\left[\gamma_{c}, \gamma_{d}\right]$. Spin-connection $\omega_{\mu}^{c d}$ should be determined by the equation obtained from the variation of the full action with respect to $\omega_{\mu}^{c d}$ (see Appendix F).

¿From (E9) and using the equations of motion derived from the action $(\mathrm{E} 8),(\mathrm{E} 9)$, we get

$$
V_{a}^{\mu} \frac{\partial L_{f}}{\partial V_{a}^{\mu}}-2 L_{f}=\bar{\Psi} \Psi U^{\prime}-2 U
$$

where $U^{\prime}$ is the derivative of $U$ with respect to its argument $\bar{\Psi} \Psi$. We see that the constraint (E7) is satisfied on the mass shell (since the fermion equations of motion are used) with $M=0$ for $L_{f}$ defined by eq. (E9) if, for example, $U=c(\bar{\Psi} \Psi)^{2}$. Any other quartic interaction, like $\bar{\Psi} \gamma_{a} \Psi \bar{\Psi} \gamma^{a} \Psi, \bar{\Psi} \sigma_{a b} \Psi \bar{\Psi} \sigma^{a b} \Psi,\left(\bar{\Psi} \gamma_{5} \Psi\right)^{2}$, etc. would also satisfy the constraint (E7) on the mass shell with $M=0$. In particular, the Nambu - Jona-Lasinio model [18] would also satisfy the constraint $(\mathrm{E} 7)$ on the mass shell with $M=0$.

In the presence of Dirac fermions with the Lagrangian density (E9), the LES (29), (30) is appropriately generalized to

$$
\begin{gathered}
V_{\mu}^{a}(x)=J^{-1 / 2}(x) V_{\mu}^{\prime a}(x) ; \quad V_{a}^{\mu}(x)=J^{1 / 2}(x) V_{a}^{\prime \mu}(x) \\
\Phi(x)=J^{-1}(x) \Phi^{\prime}(x) \\
\psi(x)=J^{1 / 4}(x) \psi^{\prime}(x) ; \quad \bar{\psi}(x)=J^{1 / 4}(x) \bar{\psi}^{\prime}(x)
\end{gathered}
$$

provided that $V(\bar{\psi} \psi)$ has a form of one of the mentioned above quartic interactions. Notice that in this case the condition for the invariance of the action with the matter Lagrangian (E9) under the transformations (E11)-(E13) is not just the simple homogeneity of degree 1 in $g^{\mu \nu}$ or degree 2 in $V_{a}^{\mu}$, because of the presence of the fermion transformation (E13). 


\section{APPENDIX F: CONNECTION IN THE VSC-FORMALISM}

We analyze here what is the dependence of the spin connection $\omega_{\mu}^{a b}$ on $V_{\mu}^{a}, \chi, \Psi$ and $\bar{\Psi}$. Varying the action (76) with respect to $\omega_{\mu}^{a b}$ and making use that

$$
R(V, \omega) \equiv-\frac{1}{4 \sqrt{-g}} \varepsilon^{\mu \nu \alpha \beta} \varepsilon_{a b c d} V_{\alpha}^{c} V_{\beta}^{d} R_{\mu \nu}^{a b}(\omega)
$$

we obtain

$$
\varepsilon^{\mu \nu \alpha \beta} \varepsilon_{a b c d}\left[\chi V_{\alpha}^{c} D_{\nu} V_{\beta}^{d}+\frac{1}{2} V_{\alpha}^{c} v_{\beta}^{d} \chi,{ }_{\nu}\right]+\frac{\kappa}{4} \sqrt{-g} V^{c \mu} \varepsilon_{a b c d} \bar{\Psi} \gamma^{5} \gamma^{d} \Psi=0
$$

where

$$
D_{\nu} V_{a \beta} \equiv \partial_{\nu} V_{a \beta}+\omega_{\nu a}^{d} V_{d \beta}
$$

The solution of Eq. ( $\mathrm{F2}$ ) is represented in the form

$$
\omega_{\mu}^{a b}=\omega_{\mu}^{a b}(V)+K_{\mu}^{a b}(\sigma)+K_{\mu}^{a b}(V, \bar{\Psi}, \Psi)
$$

where

$$
\omega_{\mu}^{a b}(V)=V_{\alpha}^{a} V^{b \nu}\left\{\begin{array}{c}
\alpha \\
\mu \nu
\end{array}\right\}-V^{b \nu} \partial_{\mu} V_{\nu}^{a}
$$

is the Riemannian part of the connection,

$$
K_{\mu}^{a b}(\sigma)=\frac{1}{2} \sigma_{, \alpha}\left(V_{\mu}^{a} V^{b \alpha}-V_{\mu}^{b} V^{a \alpha}\right)
$$

and

$$
K_{\mu}^{a b}(V, \bar{\Psi}, \Psi)=\frac{\kappa}{8} \eta_{c i} V_{d \mu} \varepsilon^{a b c d} \bar{\Psi} \gamma^{5} \gamma^{i} \Psi
$$

Notice that the spin-connection $\omega_{\mu}^{a b}$ defined by Eqs. (F4)-(F4) is invariant under the LES transformations (E11)-(E13).

[1] I. Novikov, Evolution of the Universe, Cambridge University Press, 1983. S.Weinberg, Rev. Mod. Phys. 61, 1 (1989); Y.J. Ng, Int. J. Mod. Phys. D1, 145, (1992); Gravitation and Modern Cosmology, The Cosmological Constant Problem, edited by A.Zichichi, V. deSabbata and N. Sanchez (Ettore Majorana International Science Series, Plenum Press, 1991). M. Veltman, "Reflections on the Higgs system". Preprint CERN 97-05.

[2] E.I. Guendelman and A.B. Kaganovich, Phys. Rev. D53, 7020 (1996). See also in:Proceedings of the third Alexander Friedmann International Seminar on Gravitation and Cosmology, edited by Yu.N.Gnedin, A.A. Grib, V.M. Mostepanenko (Friedman Laboratory Publishing, St Petersburg, 1995).

[3] E.I. Guendelman and A.B. Kaganovich, Phys. Rev.D55, 5970 (1997); E.I. Guendelman and A.B. Kaganovich, Mod. Phys. Lett. A12, 2421 (1997).

[4] E.I. Guendelman and A.B. Kaganovich, Phys. Rev.D56, 3548 (1997). E.I. Guendelman and A.B. Kaganovich, Hadronic Journal 21, 19 (1998).

[5] E.I. Guendelman and A.B. Kaganovich, Mod. Phys. Lett. A13, 1583 (1998).

[6] F. Gronwald, U. Muench and F. W. Hehl, Hadronic Journal, 21, 3 (1998).

[7] E.I. Guendelman and A.B. Kaganovich, Phys. Rev. D57, 7200 (1998).

[8] E.I. Guendelman and A.B. Kaganovich, "Gravity, Cosmology and Particle Fields Dynamics without the Cosmological Constant Problem", to appear in the Proceedings of the sixth International Symposium on Particles, Strings and Cosmology, PASCOS-98. 
[9] E.I. Guendelman and A.B. Kaganovich, "Field Theory Models without the Cosmological Constant Problem", Plenary talk (given by E. I. Guendelman) on the fourth Alexander Friedmann International Seminar on Gravitation and Cosmology; gr-qc/9809052.

[10] F. W. Hehl, J. D. McCrea, E. W. Mielke, Yuval Ne'eman, Phys. Reports, 258,1 (1995).

[11] A.Einstein, The Meaning of Relativity, Fifth Edition, MJF Books, N.Y.1956 ( see Appendix II).

[12] A. H. Guth, Phys. Rev. D23, 347 (1981); A. D. Linde, Phys. Lett. 108B, 389 (1982); A. Albrecht and P. J. Steinhardt, Phys. Rev. Lett. 48, 1220 (1982), A. A. Starobinsky, Phys. Lett. B91, 99 (1980); A. D. Linde, Phys. Lett. B129, 177 (1983); D. La and D. J. Steinhardt, Phys. Rev. Lett. 62, 376 (1989).

[13] See, for example: E. Kolb and M. S. Turner, The Early Universe, Addison Wesley, 1990 (see p.314).

[14] E.I. Guendelman, "Scale invariance, new inflation and decaying Lambda terms", gr-qc/9901017, to appear in Mod. Phys. Lett. A.

[15] E.I. Guendelman, "Scale invariance, mass and cosmology", gr-qc/9901067.

[16] E.I. Guendelman, Phys. Lett. B412, 42 (1997)

[17] See for example, P.G.O.Freund, Introduction to Supersymmetry, Cambridge University Press, 1986, Chapt.21; V.de Sabbata and M.Gasperini, Introduction to Gravitation, World Scientific (1985); B.de Wit and D.Z.Freedman, "Supergravity-The basics and beyond", MIT preprint CPT N1238, January 1985.

[18] Y.Nambu and G.Jona-Lasinio, Phys. Rev. 122, 345 (1961).

[19] Y. Nambu, Proceedings of the 1988 Kazimierz Workshop, Z. Ajduk et al., eds. (World Scientific, 1989); Proceedings of the 1988 Nagoya Workshop, M. Bando et al., eds. (World Scientific, 1989). W.Bardeen, C. Hill and M.Linder, Phys. Rev. D41, 1647 (1990)

[20] L. B. Okun, Leptons and Quarks, Amsterdam, North Holland, 1982.

[21] S. Coleman, Aspects of Symmetry, Cambridge Univ. Press, Cambridge,1985.

[22] S. Coleman, Nucl. Phys. B310, 643 (1988); J. Preskill, ibid. B323, 141 (1989); G. B. Giddings and A. Strominger, ibid., B307, 854 (1988); S. W. Hawking, ibid., B335, 155 (1990).

[23] The early works on this subject are: G. K. Savvidy, Phys. Lett. B71, 133 (1977); N. K. Nielsen and P. Olesen, Nucl. Phys. B144, 376 (1978); N. K. Nielsen and P. Olesen, Phys. Lett. B79, 304 (1978).

[24] E. I. Guendelman, E. Nissimov, S. Pacheva, Phys. Lett., B360, 57 (1995).

[25] C. Castro, Int. Journ. of Mod. Phys., A13, 1263 (1998).

[26] E. I. Guendelman, "Gauge condensates and gauge dynamics, the cosmological and strong CP problem", to appear in Int. J. Mod. Phys.A.

[27] J. A. Schouten, Ricci-Calculus, Springer-Verlag, Berlin, 1954. P. Baekler, F. W. Hehl and E. W. Mielke, "Nonmetricity and torsion: Facts and Fancies in gauge approaches to gravity", in: Procc. of the Fourth Marcel Grossman Meeting on General Relativity, ed. by R. Rufini, Elsevier Sc., 1986.

[28] A. Aurilia, H. Nicolai and P. K. Townsend, Nucl.Phys. B176, 509 (1980); S. W. Hawking, Phys. Lett., 134B, 276 (1984); E. Witten, in Shelter II 1985 Proc. 1983 Shelter Island Conference on Quantum Field Theory and the Fundamental Problems of Physics (Cambridge, MA: MIT Press).

[29] A. Aurilia, D. Christodoulou and F. Legovini, Phys. Lett. 73B, 429 (1978); A. Aurilia, G. Denardo, F. Legovini and E. Spalluci, ibid., 147B, 258 (1984); J. D. Brown and C. Teitelboim, Phys. Lett. 195B, 177 (1987); Nucl. Phys. B297, 787 (1988).

[30] Y. Ne'eman, Ann. Inst. Henri Poincare XXVIII, 369 (1978); Y. Ne'eman and Dj.Sijacki, Ann. Phys. 129, 292 (1979). 